\title{
An integrated theory of deciding and acting
}

\author{
Mathieu Servant ${ }^{1 *}$, Gordon D. Logan ${ }^{2}$, Thibault Gajdos ${ }^{3}$, Nathan J. Evans ${ }^{4}$ \\ ${ }^{1}$ Laboratoire de Recherches Intégratives en Neurosciences et Psychologie Cognitive UR 481 \\ et MSHE Ledoux USR 3124, Université de Franche-Comté, France \\ ${ }^{2}$ Department of Psychological Sciences, Vanderbilt University, USA \\ ${ }^{3}$ Laboratoire de Psychologie Cognitive UMR 7286, Aix-Marseille Université, France \\ ${ }^{4}$ School of Psychology, University of Queensland, Australia
}

*Contact Information: mathieu.servant@univ-fcomte.fr

\begin{abstract}
Author Note
The empirical data, codebook, and JASP files for statistical analyses are available on the Open Science Framework (https://osf.io/r5c2b/; Servant, 2021). The authors wish to thank Boris Burle for helpful comments on an earlier version of this manuscript and Laure Spieser for technical assistance with electromyography. The ideas and data appearing in this article have been presented at the Virtual Psychonomics 2020 Annual Meeting. MS was supported by an ANER grant from the Bourgogne Franche-Comté region. NJE was supported by an Australian Research Council Discovery Early Career Researcher Award (DE200101130). GDL, TG and NJE share the senior authorship. The authors declare no conflict of interest. Correspondence concerning this article should be addressed to Mathieu Servant, Laboratoire de Recherches Intégratives en Neurosciences et Psychologie Cognitive UR 481, UFR Santé, bâtiment Rabelais, Université de Franche-Comté, 19 rue Ambroise Paré, 25030 Besançon Cedex, France. E-mail: mathieu.servant@univ-fcomte.fr
\end{abstract}

\section{Abstract}


This article presents a theory in which motor execution in perceptual decision-making tasks is determined by the same evolving decision variable that drives response time. The theory builds upon recent insights from the neuroscience of decision-making and motor control. It is formalized as an extension of Ratcliff's diffusion model, and assumes that two thresholds operate on the evidence accumulation decision variable. The first threshold, referred to as electromyographic (EMG) threshold, marks the onset of electrical activity in the responserelevant muscle and the beginning of force production. The second threshold corresponds to the response. The theory makes several benchmark predictions. Notably, the mean duration of motor execution, as quantified by the mean latency between EMG onset and the response, should depend on the rate of evidence accumulation, and should thus increase as the perceptual difficulty of the task increases. We tested these predictions in a paradigmatic perceptual decision-making task, the random dot motion task, and recorded the EMG activity of response-relevant muscles. The behavioral and EMG data provide very strong evidence for each prediction. A final quantitative evaluation of the model showed good fits to these data. The theory resolves conflicting findings in the fields of mathematical psychology, motor control and decision neurosciences.

Keywords: Decision-making; Motor execution; Electromyography; Motor control 
Perceptual decision-making is the process of converting sensory information into goaldirected actions (e.g. deciding whether a traffic light is red or green, and acting on the car accordingly). Because adaptive behavior hinges upon the integrity of this process, it has been the object of a considerable interdisciplinary research effort over the past 50 years. Evidence accumulation models such as the diffusion model currently represent the dominant framework for understanding perceptual decision-making (Brown \& Heathcote, 2008; Forstmann et al., 2016; Gold \& Shadlen, 2007; O'Connell et al., 2018; Ratcliff et al., 2016; Ratcliff \& Smith, 2004; Smith \& Lilburn, 2020; Usher \& McClelland, 2001). These models build upon three sequential processing stages: sensory encoding, decision-making, and motor execution. The decision stage is thought to involve accumulator units, which integrate noisy sensory information over time until a criterial amount of evidence is attained. Each accumulator is associated to a particular response alternative, and the accumulator that wins the race determines which response is chosen, and when it is chosen. This accumulation-tobound mechanism is followed by a motor process that executes the selected response.

Evidence accumulation models assume selective influence of experimental manipulations on information processing components, the latter being represented by parameters. For example, the diffusion model for two-choice perceptual decisions has four main parameters (Figure $1 \mathrm{~A})$. The average rate of evidence accumulation, called drift rate $(v)$, depends on the quality of sensory information and the efficiency of attentional processes. Decision thresholds ( $a$ : correct choice, $-a$ : incorrect choice) regulate the speed-accuracy policy of the decision. A larger separation between thresholds produces slower but more accurate responding. The starting point $(z)$ of the accumulation process reflects a potential a priori bias towards a particular response alternative. Finally, the non-decision time parameter (Ter) 
quantifies the mean duration of processes outside the decision process, such as sensory encoding and motor execution (Ratcliff, 1978; Ratcliff et al., 2016; Ratcliff \& McKoon, 2008; Ratcliff \& Rouder, 1998; Ratcliff \& Smith, 2004). This mapping between model parameters and information processing components has given rise to four sets of predictions (e.g., Ratcliff \& McKoon, 2008; Smith \& Lilburn, 2020; Voss et al., 2004). First, manipulations of perceptual difficulty should selectively modulate the drift rate parameter. Second, manipulations of speed-accuracy tradeoff (SAT) should selectively modulate threshold separation. Third, manipulations of response bias should selectively modulate the starting point of the evidence accumulation process. Finally, manipulations of sensory- or motor-related variables (e.g., the force required to press a response button) should selectively modulate mean non-decision time.

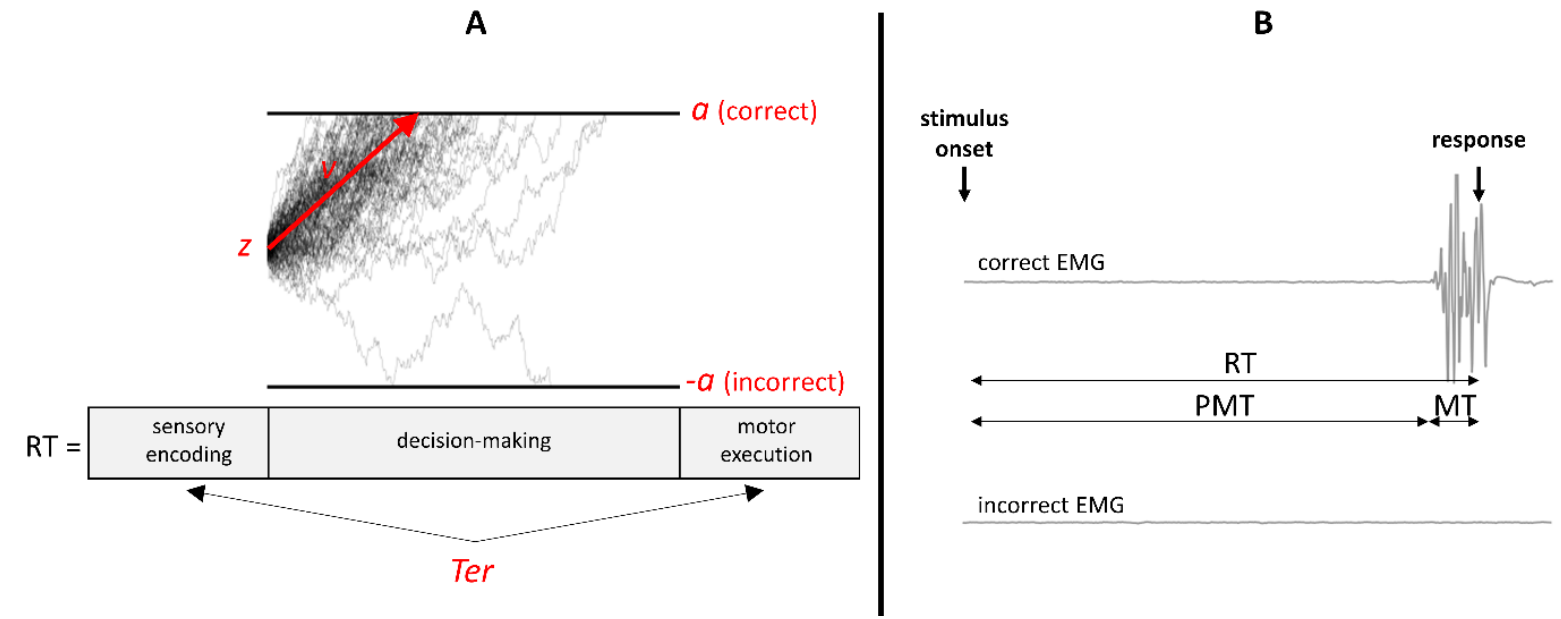

Figure 1. A) Architecture of the standard diffusion model. Model parameters are shown in red: $z=$ starting point of the evidence accumulation process, $a=$ upper decision threshold, $v=\mathrm{drift}$ rate, $\mathrm{Ter}=$ mean non-decision time. See text for details. B) Typical EMG activity in a correct trial of a perceptual decision-making task. Response time (RT) can be divided into a premotor time (PMT; from stimulus onset to the EMG onset of the response) and a motor time (MT; from EMG onset to the response). 
Several studies have been conducted to evaluate each of these predictions, yielding mixed results (for a review, see Smith \& Lilburn, 2020). Although investigators generally observed the predicted parametric modulations, some experimental manipulations modulated additional parameters. For example, manipulations of SAT have produced additional modulations of drift rate (Donkin et al., 2011; Heathcote \& Love, 2012; Ho et al., 2012; Rae et al., 2014; Starns et al., 2012) and non-decision time (Arnold et al., 2015; de Hollander et al., 2016; Huang et al., 2015; Servant et al., 2018), and these violations seem to be model-dependent (Evans, 2020). Dutilh et al. (2019) recently conducted a blinded collaborative assessment of the quality of inferences from evidence accumulation model analyses. They manipulated perceptual difficulty, SAT, and response bias in a random dot motion task. The data from 20 participants were sent to a sample of 17 teams of researchers with expertise in evidence accumulation models. Researchers were blind to the experimental manipulations, and were invited to infer the processing components (model parameters) that were modulated by the experimental manipulations by fitting an evidence accumulation model to data. In general, researchers identified the psychological processing components hypothetically modulated by each experimental manipulation. However, many researchers reported that manipulations of motion coherence, response bias, and SAT also modulated the mean non-decision time component of the model.

Because these additional parametric modulations by experimental variables were not predicted in the first place, they have been interpreted as violations of selective influence and evidence against the diffusion model architecture. However, the selective influence assumptions that have been derived from the diffusion model are not very strong. For example, drift rate is a free parameter reflecting the rate of evidence accumulation. Although the evidence may come from perceptual or memory representations, the model does not 
specify how this evidence is computed. Without this additional theoretical development, it is difficult to make precise predictions regarding what experimental variables should affect drift rate. Similarly, non-decision time is a free parameter in the model reflecting sensory encoding and motor execution latencies. The model does not specify the computations underlying sensory encoding and motor execution, and it does not specify what variables should affect the duration of these processes. A theory of sensory encoding and motor execution is required to make these predictions.

Several extensions of evidence accumulation models have been proposed to specify how drift rate is computed (e.g., Nosofsky \& Palmeri, 1997; Smith \& Lilburn, 2020; Smith \& Ratcliff, 2009; White et al., 2011). The present work focuses on the motor execution component. The next section introduces an extension of the diffusion model designed to account for a range of motor phenomena in a specific category of perceptual decision-making tasks in which a task-irrelevant attribute of the stimulus can automatically activate a response opposite to the response associated with the task-relevant stimulus attribute, thus generating a response conflict (e.g., Stroop, flanker, Simon tasks). We will then hypothesize that this theoretical extension may generalize beyond conflict tasks. To test this hypothesis, we will first derive a set of five broad qualitative predictions from the model and submit them to an empirical test. A quantitative assessment will finally be performed by fitting the model to data.

\section{A dual-threshold diffusion model for deciding and acting}

Motor execution in perceptual decision-making tasks has been studied using a variety of methodologies (for a review, see Miller et al., 1999). Among these methodologies, the electromyography (EMG) of response-relevant muscles (e.g., the flexor pollicis brevis for a button press with the thumb) appears particularly valuable because it allows for an analysis 
of motor execution on a trial-to-trial basis (Burle et al., 2002; Burle \& Bonnet, 1999; e.g., Coles et al., 1985; Hasbroucq et al., 2001; Rochet et al., 2014; Servant et al., 2015; Spieser et al., 2017; Weindel et al., 2020). EMG measures the myoelectric activity of muscle fibers in response to neural excitation (Stålberg et al., 2019; Vigotsky et al., 2018), which initiates force production after an electromechanical delay (Disselhorst-Klug et al., 2009; Perry \& Bekey, 1981). Figure 1B illustrates the typical EMG activity of response agonists in a correct trial of a perceptual decision-making task involving a choice between two manual responses. Thanks to the excellent signal-to-noise ratio of EMG, EMG onsets can be detected in each trial. Researchers can then divide each response time (RT) into a premotor time (PMT; from stimulus onset to the EMG onset of the response) and a motor time (MT; from EMG onset to the response). MT is a measure of the execution of the response, while PMT is determined by upstream processes such as perception and decision-making.

The above RT fractionation technique based on EMG has been used to evaluate the effect of several experimental factors on premotor and motor execution processes. A summary of findings obtained with a set of common experimental manipulations in perceptual decision-making tasks (stimulus-response compatibility, perceptual difficulty, SAT, response bias, response force) is provided in Table 1. The bulk of studies has been conducted in conflict tasks for two reasons. First, investigators wanted to determine whether the stimulus-response compatibility factor modulates both mean PMT and mean MT. Compatible trials correspond to trials in which relevant and irrelevant stimulus attributes activate the same response (e.g., the word RED printed in red in a Stroop task). Incompatible trials correspond to trials in which relevant and irrelevant stimulus attributes activate opposite responses (e.g., the word RED printed in blue in a Stroop task). The majority of studies reported a significant modulation of mean PMT only. Second, EMG signals in conflict tasks sometimes exhibit a surprising 
phenomenon in correct trials. This phenomenon, termed partial error, takes the form of a small covert EMG burst in the incorrect response channel that occurs before the EMG onset of the correct response (Figure 2B). The proportion of partial errors is systematically higher in the incompatible compared to the compatible condition, suggesting that partial errors do not simply reflect motor noise.

The very existence of partial errors requires a theory explaining how, why, and when discrete motor events sometimes occur during the decision process. The dual-threshold diffusion model (DTDM; Servant et al., 2015) for deciding and acting has been developed for this purpose. The core assumption of DTDM is that EMG activity is determined by the same evolving decision variable that drives RT. This hypothesis appears plausible in light of the neurophysiology of the musculoskeletal motor system. Electrophysiological studies in perceptual decision-making tasks have shown that the primary motor cortex (M1) exhibits choice predictive activities that reflect integrated sensory evidence (e.g., Afacan-Seref et al., 2018; Donner et al., 2009; Gould et al., 2012; Kelly \& O'Connell, 2013; O'Connell et al., 2012, 2018; Servant et al., 2016; Steinemann et al., 2018; Wyart et al., 2012). On average, the electrical activity over M1 contralateral to the response reaches a peak amplitude after EMG onset (Servant et al., 2016), suggesting that evidence accumulation carries on after a command has been sent to the response-relevant muscle. Although M1 neurons do not form synapses with muscles, the most direct pathway for muscle activation involves a monosynaptic connection in the spinal cord between the axons of M1 pyramidal tract neurons and alpha motoneurons that innervate muscles (Bernhard \& Bohm, 1954; Ebbesen \& Brecht, 2017). These direct cortico-motorneuronal connections are relatively recent in the evolutionary history, and appear to be involved in fine motor behaviors involving the distal extremities (e.g., independent finger movements such as those required for a button press; 
Lemon, 2008). Consequently, the decision variable may be transmitted to the muscles without undergoing significant transformations. Strong support for this hypothesis comes from a study in which participants performed a perceptual decision-making task and indicated their decision by moving a handle of a robotic manipulandum to one of two targets (Selen et al., 2012). During decision formation, the state of the peripheral motor system was probed by briefly extending the elbow at random times, thereby eliciting a stretch reflex. The reflex gain, as quantified by EMG, reflected the accumulated sensory evidence in support of the evolving decision.

The general architecture of the DTDM is similar to that of the standard diffusion model, with one important difference. The decision threshold $a$ does not indicate a commitment to a choice; it simply marks the onset of EMG activity. To emphasize this functional difference, we refer to this threshold as EMG threshold (parameter $m$ ), and assume that it operates at the M1 level. DTDM explicitly assumes that evidence accumulation continues until a response (button press) is made, and a second threshold (parameter $r$ ) corresponds to the response. Two thresholds thus operate on the decision variable: the EMG threshold (-m: EMG activity in the incorrect response channel, $m$ : EMG activity in the correct response channel), and the response threshold (-r: incorrect response; $r$ : correct response), with $|m|<|r|$. In this framework, PMT corresponds to the latency at which the decision variable hits the EMG threshold $m$, and MT corresponds to the latency at which the decision variable hits the response threshold $r$ minus PMT (Figure 2A). In addition, PMT and MT incorporate residual (nondecisional) processing components with mean duration $T e$ and $\operatorname{Tr}$ respectively, though the precise nature of these components is unclear. At minimum, Te incorporates a sensory encoding latency and the corticomuscular transmission time. $T r$ incorporates the electromechanical delay, defined as the latency between muscle excitation and force 
production (Cavanagh \& Komi, 1979; Lacourpaille et al., 2013). The electromechanical delay comprises both electrochemical and mechanical processes involved in force production (e.g., cross-bridge formation between actin and myosin filaments).

Metaphorically, the transmission of information between decision-making and motor execution can be conceptualized as a flow of water (the decision variable) regulated by a faucet (EMG threshold). When the decision variable hits the EMG threshold, the brain turns on the faucet, and the water activates the muscle, producing force. The response will be given if a sufficient amount of water (response threshold) is discharged. However, if the decision variable goes below the EMG threshold before having reached the response threshold, the brain will turn off the faucet, resulting in a partial EMG burst (generating insufficient force to produce a response). DTDM thus predicts a partial EMG burst when the decision variable hits an EMG threshold but goes back in the space delimited by the two EMG thresholds. Consequently, DTDM predicts a partial error when the decision variable hits the incorrect EMG threshold $-m$ but finally reaches the correct response threshold $r$ (Figure 2B). If compatible and incompatible trials had the same drift rate, the model would predict the same partial error rate in both conditions. However, diffusion models of conflict tasks typically assume a timevarying drift rate, whereby the decision evidence is dominated by the irrelevant stimulus attribute in early stages of processing, and becomes progressively determined by the relevant stimulus attribute only (Hübner et al., 2010; Ulrich et al., 2015; White et al., 2011). With this additional time-varying drift rate assumption, Servant et al. (2015) demonstrated that DTDM predicts the rate, latency, and correction time of partial errors in both compatible and incompatible trials. In addition, the model generally predicts no effect of compatibility on mean MT, consistent with observed data, because the drift rate of incompatible trials converges towards the drift rate of compatible trials as processing time increases. 
DTDM is appealing because it provides a theoretical account of both decision-making and motor execution in conflict tasks. Thus, the main goal of this article is to determine whether DTDM can generalize beyond conflict tasks, and predict EMG data in standard perceptual decision-making tasks as well (in which performance is only determined by taskrelevant stimulus information). A core assumption of DTDM is that EMG activity is determined by the evolving decision variable. Consequently, experimental manipulations designed to modulate drift rate, such as manipulations of perceptual difficulty, should modulate mean PMT as well as mean MT. Specifically, an increase in perceptual difficulty should decrease drift rate, and should thus increase both mean PMT and mean MT. To the best of our knowledge, six experiments have examined the effect of perceptual difficulty on mean PMT and mean MT in perceptual decision-making tasks (see Table 1). In general, these studies reported significant increases of both mean PMT and mean MT as perceptual difficulty increases in both conflict and non-conflict tasks, but the absolute effect sizes are very small (up to $8 \mathrm{~ms}$ ). In addition, two experiments reported a significant modulation of mean PMT only (Miller et al., 1999).

There are at least two explanations for these somewhat equivocal findings. First, the force required to issue the response greatly varies between studies, and has often not been reported. This parameter is important within DTDM because it determines the distance (in accumulation units) between EMG and response thresholds. If the force required to respond is low (e.g., 0.44-0.59 $\mathrm{N}$ for a typical computer keyboard), the distance between EMG and response thresholds will be small. Consequently, MT will be short, and modulations of drift rate will produce small modulations of mean MT that can be missed if the sampling rate of EMG signals is low or if signals are noisy. Conversely, if the force required to respond is high (e.g., the thermoplastic rubber hand-held buttons of the Black Box Toolkit module ${ }^{\mathrm{TM}}$ used by several psychology laboratories, $\sim 8.83 \mathrm{~N}$ ), the distance between EMG and response thresholds 
will be larger. Consequently, the same modulations of drift rate will produce larger effects on mean MT. Second, Table 1 shows that some perceptual difficulty manipulations produced a small effect on mean PMT (e.g., 30 ms for the two experiments of Miller et al. 1999). Small modulations of drift rate will produce small modulations of MT that can be hard to detect, especially if the force required to respond is low.

In order to provide a rigorous test of the assumption -core to DTDM- that EMG activity is determined by the evolving decision variable, a large modulation of drift rate coupled with a relatively high force required to respond is necessary. For this reason, we manipulated perceptual difficulty in a random dot motion task (RDM), and used the thermoplastic rubber hand-held buttons of the Black Box Toolkit module ${ }^{\mathrm{TM}}(8.83 \mathrm{~N})^{1}$. RDM requires participants to determine the global direction of a random dot kinematogram featuring a proportion of dots moving coherently in the left or right signal direction (Figure 3A). This proportion, termed motion coherence, determines the perceptual difficulty of the decision. Specifically, perceptual difficulty increases as motion coherence decreases. Coherence manipulations can produce effects on RT much larger in magnitude (726 ms in the upcoming experiment) compared to tasks in which all the evidence appears at a given onset time, such as those used by previous EMG studies (up to $110 \mathrm{~ms}$, see Table 1). Consequently, modulations of drift rate in our RDM task are expected to be large. DTDM makes several EMG predictions that are detailed in the next section.

\footnotetext{
${ }^{1}$ Although we wanted to manipulate the force required to respond in this experiment, the lab of the first author is not currently equipped with force sensors.
} 


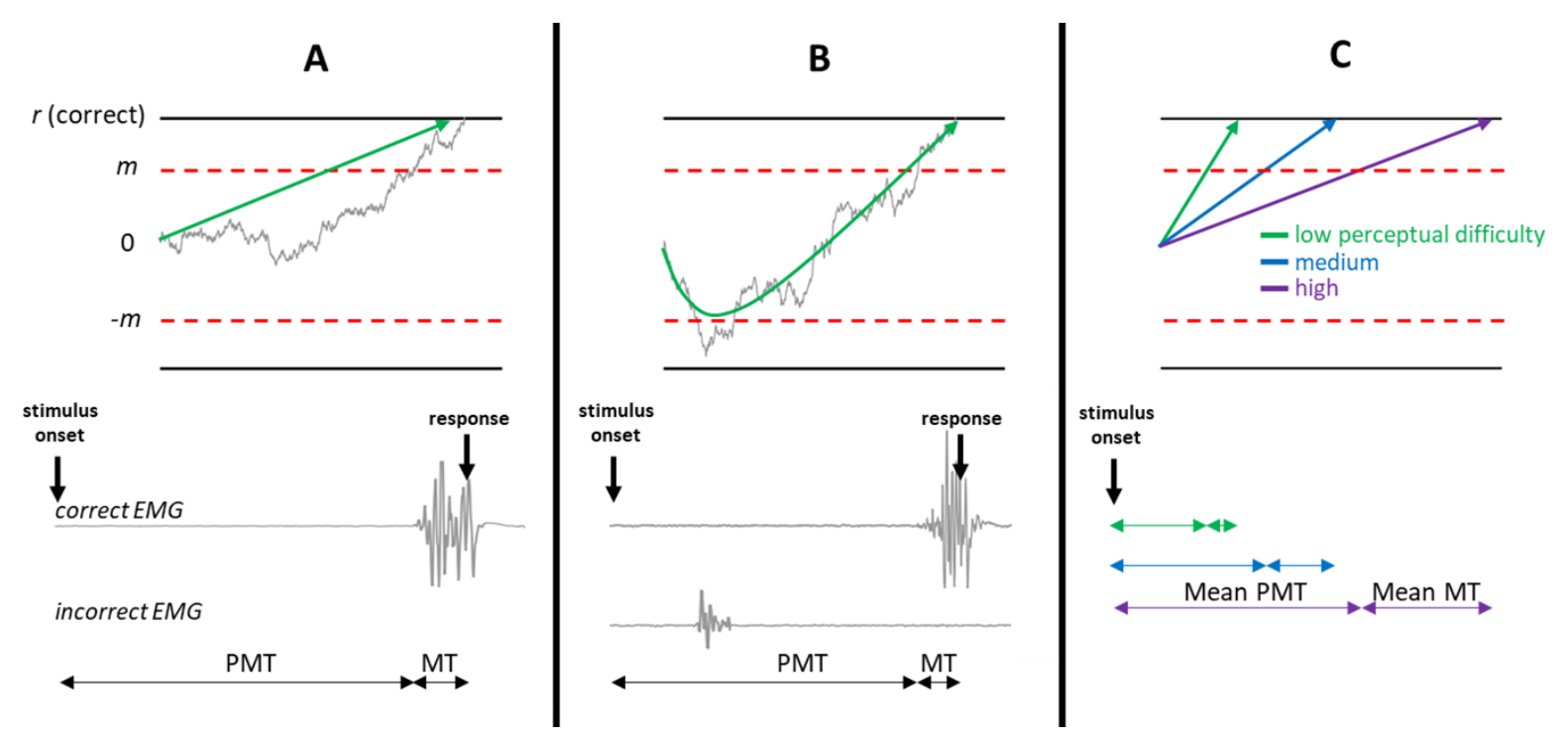

Figure 2. Architecture of the dual-threshold diffusion model (DTDM) for deciding and acting and EMG predictions. A) DTDM assumes that EMG activity is determined by the same evolving decision variable that drives RT. Two different types of thresholds thus operate on the decision variable. EMG thresholds $( \pm m)$ determine the level of accumulated evidence required to transmit information to the response-relevant muscle and generate EMG activity. Response thresholds $( \pm r$ ) determine the level of accumulated evidence required to issue a response. The noisy sample path represents the trajectory of the decision variable in a given trial of a perceptual decision-making task and the green straight line represents the accumulated drift rate. The predicted PMT corresponds to the latency at which the decision variable hits the EMG threshold $m$, and the predicted MT corresponds to the latency at which the decision variable hits the response threshold $r$ minus PMT. PMT and MT also incorporate residual (nondecisional) processing components, with mean duration $\mathrm{Te}$ and $\mathrm{Tr}$ respectively (not shown for sake of clarity). B) Typical partial error trial in the incompatible condition of a conflict task. Although the response is correct, a small partial EMG burst, called partial error, develops in the incorrect EMG channel during PMT. DTDM predicts a partial error when the decision variable hits the incorrect EMG threshold $-m$ but finally reaches the correct response threshold $r$. The green line represents the accumulated time-varying drift rate assumed by diffusion models of conflict tasks in the incompatible condition. On average, the decision evidence is dominated by the irrelevant stimulus attribute in early stages of processing, and becomes progressively determined by the relevant stimulus attribute only. This property increases the likelihood of partial errors in the incompatible condition. C) Predicted mean PMT and mean MT in a standard perceptual decision-making task featuring different levels of perceptual difficulty, such as the random dot motion task. Because EMG activity is determined by the decision variable, mean PMT and mean MT are determined by the drift rate. Consequently, mean PMT and mean MT should increase as perceptual difficulty increases. 


\section{DTDM predictions}

(1) The first prediction, derived from the assumption that EMG is determined by the decision variable, is that both mean PMT and mean MT should monotonically increase as motion coherence decreases. This prediction is illustrated in Figure 2C.

(2) EMG bursts are characterized by a sequence of depolarizations and hyperpolarizations that occur on the muscle fiber membrane (see Figures $1 \mathrm{~B}, 2 \mathrm{~A}$, and $2 \mathrm{~B}$ ), so averaging these bursts would lead to a signal close to zero. To analyze muscle excitation, researchers generally take the absolute value of voltages across time points, a procedure known as rectification (Vigotsky et al., 2018). A rectified EMG burst has a sudden onset (similar to a raw burst), and then exhibits a gradual and noisy build-up of activity followed by a decline back to the baseline electrical level. An illustration of the rectification procedure is provided in Supplementary Figure S1. DTDM predicts that the gradual build-up of rectified and averaged EMG bursts should reflect the accumulated drift rate, and should thus monotonically decrease as coherence decreases.

(3) Because evidence accumulation is a noisy process, the decision variable can sometimes oscillate around the EMG threshold before reaching the response threshold. This would produce partial EMG bursts during PMT (similar to partial errors in conflict tasks). Consequently, DTDM predicts partial EMG bursts during PMT, and the proportion of trials featuring partial bursts should increase as motion coherence decreases, because there is less evidence (motion energy) to drive the decision variable to the response threshold. For the same reason, the mean latency of the first partial EMG burst should increase as motion coherence decreases. Two recent studies have observed partial EMG bursts in standard perceptual decision-making tasks (Gabor orientation, Gajdos et al., 2019; contrast 
discrimination, Weindel et al., 2020). Although one of these studies manipulated perceptual difficulty (Weindel et al., 2020), the effect of perceptual difficulty on partial EMG bursts has not been analyzed.

(4) The standard diffusion model predicts right-skewed RT distributions that have the same shape as those observed in perceptual decision-making tasks (Ratcliff, 1978; Ratcliff et al., 2016; Ratcliff \& McKoon, 2008). Consequently, DTDM predicts that the distributions of PMT and MT should exhibit a similar right-skewed shape for each subject and motion coherence level, which should translate into an approximately linear PMT quantile MT quantile (QQ) plot (Supplementary Figure S2A).

(5) Without between-trial variability in the rate of evidence accumulation, DTDM predicts a null between-trial correlation between PMT and MT within each coherence level. This assumption stems from the Markov (memoryless) property of the diffusion process: the conditional probability distribution of future states of the process only depends upon the present state (Supplementary Figure S2B). However, the predicted between-trial correlation between PMT and MT necessarily increases as between-trial variability in drift rate increases (Supplementary Figure S3). A modest amount of between-trial variability in drift rate is usually incorporated into the DDM, because internal representations of the same stimulus presumably vary from trial to trial. It also allows the model to predict slower error than correct responses, a pattern generally observed when decision-making is difficult (Ratcliff, 1978; Ratcliff et al., 2016; Ratcliff \& McKoon, 2008). Consequently, we anticipated a small positive correlation between PMT and MT in our random dot motion task. To the best of our knowledge, only one study analyzed the between-trial correlation between PMT and MT in a perceptual decision-making task (contrast discrimination; Weindel et al., 2020). Interestingly, 
this study reported a null correlation, consistent with DTDM with no between-trial variability in drift.

Table 1. Summary of EMG studies examining the effect of a set of common experimental manipulations in perceptual decision-making tasks (sub-tables: stimulus-response compatibility, perceptual difficulty, speed-accuracy tradeoff, response bias, response force) on PMT and MT. Within each sub-table, studies are organized in chronological order.

\begin{tabular}{ccccc}
\hline $\begin{array}{c}\text { Experiment } \\
\text { manipulation and }\end{array}$ & Statistically & Statistically & Statistically & Muscle \\
corresponding studies & significant & significant & significant & recorded \\
& modulation of & modulation of MT? & modulation of & and force \\
& PMT? & & required to \\
issue the \\
\end{tabular}

\begin{tabular}{|c|c|c|c|c|}
\hline $\begin{array}{l}\text { Eriksen et al. (1985) } \\
\text { flanker task }\end{array}$ & $\begin{array}{c}\text { Yes } \\
\text { PMT compatible < } \\
\text { PMT incompatible }\end{array}$ & $\begin{array}{c}\text { Yes } \\
\text { MT compatible < } \\
\text { MT incompatible }\end{array}$ & $\begin{array}{c}\text { Yes } \\
\text { ER compatible }(M= \\
5.0 \%)< \\
\text { ER incompatible }(M= \\
15.0 \%)\end{array}$ & $\begin{array}{l}\text { Forearm } \\
\text { flexor }\end{array}$ \\
\hline $\begin{array}{l}\text { Coles et al. (1985) } \\
\text { flanker task }\end{array}$ & $\begin{array}{c}\text { Yes } \\
\text { PMT compatible < } \\
\text { PMT incompatible }\end{array}$ & $\begin{array}{c}\text { Yes } \\
\text { MT compatible < } \\
\text { MT incompatible }\end{array}$ & Yes & $\begin{array}{l}\text { Forearm } \\
\text { flexor, } 25 \% \text { of } \\
\text { maximum } \\
\text { force }\end{array}$ \\
\hline $\begin{array}{l}\text { Smid et al. (1990) } \\
\text { flanker task }\end{array}$ & $\begin{array}{c}\text { Yes } \\
\text { PMT compatible }(M= \\
316 \mathrm{~ms})<\text { PMT } \\
\text { incompatible }(M= \\
359 \mathrm{~ms})\end{array}$ & $\begin{array}{c}\text { Yes } \\
\text { MT compatible }(M= \\
112 \mathrm{~ms})<\mathrm{MT} \\
\text { incompatible }(M=118 \\
\mathrm{ms})\end{array}$ & $\begin{array}{c}\text { Yes } \\
\text { ER compatible }(M= \\
1.8 \%)< \\
\text { ER incompatible }(M= \\
8.1 \%)\end{array}$ & $\begin{array}{c}\text { Flexor } \\
\text { digitorum } \\
2.94 \mathrm{~N} \text { force }\end{array}$ \\
\hline $\begin{array}{c}\text { Hasbroucq et al. (1999) } \\
\text { Simon task }\end{array}$ & $\begin{array}{c}\text { Yes } \\
\text { PMT compatible }(M= \\
244 \mathrm{~ms})<\text { PMT } \\
\text { incompatible }(M= \\
261 \mathrm{~ms})\end{array}$ & $\begin{array}{c}\text { No } \\
\text { MT compatible: } \\
M=126 \mathrm{~ms} \\
\text { MT incompatible: } \\
M=129 \mathrm{~ms}\end{array}$ & $\begin{array}{c}\text { No } \\
\text { ER compatible: } \\
M=3.5 \% \\
\text { ER incompatible: } \\
M=4.6 \%\end{array}$ & $\begin{array}{c}\text { Flexor pollicis } \\
\text { brevis } \\
9.81 \mathrm{~N} \text { force }\end{array}$ \\
\hline $\begin{array}{l}\text { Burle and Bonnet (1999) } \\
\text { Simon task }\end{array}$ & $\begin{array}{c}\text { Yes } \\
\text { PMT compatible }(M= \\
237 \mathrm{~ms})<\mathrm{PMT} \\
\text { incompatible }(M= \\
257 \mathrm{~ms})\end{array}$ & No & No & $\begin{array}{c}\text { Flexor pollicis } \\
\text { brevis } \\
20 \mathrm{~N} \text { force }\end{array}$ \\
\hline $\begin{array}{c}\text { Hasbroucq et al. (2001) } \\
\text { Fitts task }\end{array}$ & $\begin{array}{c}\text { Yes } \\
\text { PMT compatible }(M= \\
183 \mathrm{~ms})<\mathrm{PMT} \\
\text { incompatible }(M= \\
216 \mathrm{~ms})\end{array}$ & $\begin{array}{c}\text { No } \\
\text { MT compatible: } \\
M=126 \mathrm{~ms} \\
\text { MT incompatible: } \\
M=130 \mathrm{~ms}\end{array}$ & $\begin{array}{c}\text { No } \\
\text { ER compatible: } \\
M=1.2 \% \\
\text { ER incompatible : } \\
M=1.3 \%\end{array}$ & $\begin{array}{c}\text { Flexor pollicis } \\
\text { brevis } \\
5.88 \mathrm{~N} \text { force }\end{array}$ \\
\hline $\begin{array}{l}\text { Burle et al. (2002) } \\
\text { Simon task }\end{array}$ & $\begin{array}{c}\text { Yes } \\
\text { PMT compatible }(M= \\
288 \mathrm{~ms})<\mathrm{PMT} \\
\text { incompatible }(M= \\
316 \mathrm{~ms})\end{array}$ & $\begin{array}{c}\text { No } \\
\text { MT compatible: } \\
M=106 \mathrm{~ms} \\
\text { MT incompatible: } \\
M=106 \mathrm{~ms}\end{array}$ & No & $\begin{array}{c}\text { Flexor pollicis } \\
\text { brevis } \\
\text { low }(0.49 \mathrm{~N}) \\
\text { versus high } \\
(9.81 \mathrm{~N}) \text { force }\end{array}$ \\
\hline $\begin{array}{l}\text { Hasbroucq et al. (2009) } \\
\text { Simon task }\end{array}$ & $\begin{array}{c}\text { Yes } \\
\text { PMT compatible }(M= \\
183 \mathrm{~ms})<\mathrm{PMT}\end{array}$ & $\begin{array}{c}\text { No } \\
\text { MT compatible: } \\
M=118 \mathrm{~ms} \\
\text { MT incompatible: }\end{array}$ & & $\begin{array}{c}\text { Flexor pollicis } \\
\text { brevis, } \\
10 \mathrm{~N} \text { force }\end{array}$ \\
\hline
\end{tabular}




\begin{tabular}{|c|c|c|c|c|}
\hline & $\begin{array}{c}\text { incompatible }(M= \\
216 \mathrm{~ms})\end{array}$ & $M=118 \mathrm{~ms}$ & & \\
\hline $\begin{array}{l}\text { Servant et al. (2015) } \\
\quad \text { flanker task }\end{array}$ & $\begin{array}{c}\text { Yes } \\
\text { PMT compatible }(M= \\
296 \mathrm{~ms})<\mathrm{PMT} \\
\text { incompatible }(M= \\
339 \mathrm{~ms})\end{array}$ & $\begin{array}{c}\text { No } \\
\text { MT compatible: } \\
M=122 \mathrm{~ms} \\
\text { MT incompatible: } \\
M=122 \mathrm{~ms}\end{array}$ & $\begin{array}{c}\text { Yes } \\
\text { ER compatible }(M= \\
2.3 \%)< \\
\text { ER incompatible }(M= \\
6.0 \%)\end{array}$ & $\begin{array}{c}\text { Flexor pollicis } \\
\text { brevis }\end{array}$ \\
\hline $\begin{array}{l}\text { Servant et al. (2016) } \\
\text { Simon task, manipulation } \\
\text { of color saturation }\end{array}$ & $\begin{array}{c}\text { Yes } \\
\text { PMT compatible }(M= \\
346 \mathrm{~ms})<\mathrm{PMT} \\
\text { incompatible }(M= \\
368 \mathrm{~ms})\end{array}$ & $\begin{array}{c}\text { No } \\
\text { MT compatible: } \\
M=133 \mathrm{~ms} \\
\text { MT incompatible: } \\
M=133 \mathrm{~ms}\end{array}$ & $\begin{array}{c}\text { Yes } \\
\text { ER compatible }(M= \\
4.8 \%)< \\
\text { ER incompatible }(M= \\
7.9 \%)\end{array}$ & $\begin{array}{c}\text { Flexor pollicis } \\
\text { brevis }\end{array}$ \\
\hline $\begin{array}{l}\text { Spieser et al. (2017) } \\
\text { flanker task }\end{array}$ & $\begin{array}{c}\text { Yes } \\
\text { PMT compatible }(M= \\
293 \mathrm{~ms})<\mathrm{PMT} \\
\text { incompatible }(M= \\
308 \mathrm{~ms})\end{array}$ & $\begin{array}{c}\text { No } \\
\text { MT compatible: } \\
M=94 \mathrm{~ms} \\
\text { MT incompatible: } \\
M=95 \mathrm{~ms}\end{array}$ & $\begin{array}{c}\text { Yes } \\
\text { ER compatible }(M= \\
13.9 \%)< \\
\text { ER incompatible }(M= \\
24.1 \%)\end{array}$ & $\begin{array}{c}\text { Flexor pollicis } \\
\text { brevis }\end{array}$ \\
\hline \multicolumn{5}{|l|}{$\begin{array}{l}\text { Perceptual } \\
\text { difficulty }\end{array}$} \\
\hline $\begin{array}{l}\text { Romaiguère et al. (1993) } \\
\text { Simon task, manipulation } \\
\text { of light intensity }\end{array}$ & $\begin{array}{c}\text { Yes } \\
\text { PMT lowest } \\
\text { perceptual difficulty } \\
\text { ( } M=383 \mathrm{~ms})<\text { PMT } \\
\text { highest perceptual } \\
\text { difficulty }(M=400 \\
\mathrm{ms})\end{array}$ & $\begin{array}{c}\text { Yes } \\
\text { MT lowest perceptual } \\
\text { difficulty }(M=313 \mathrm{~ms}) \\
<\text { MT highest } \\
\text { perceptual difficulty ( } M \\
=321 \mathrm{~ms})\end{array}$ & No & $\begin{array}{l}\text { Abductor } \\
\text { pollicis brevis } \\
\text { low ( } 2.45 \mathrm{~N}) \\
\text { versus high } \\
\text { (15.44 N) } \\
\text { force }\end{array}$ \\
\hline $\begin{array}{l}\text { Miller et al. (1999) Exp } 1 \\
\text { letter discrimination, } \\
\text { manipulation of contrast }\end{array}$ & $\begin{array}{c}\text { Yes } \\
\text { PMT lowest } \\
\text { perceptual difficulty } \\
(M=262 \mathrm{~ms})<\text { PMT } \\
\text { highest perceptual } \\
\text { difficulty }(M=291 \\
\text { ms) }\end{array}$ & $\begin{array}{l}\text { No } \\
\text { MT lowest perceptual } \\
\text { difficulty: } M=186 \mathrm{~ms} ; \\
\text { MT highest perceptual } \\
\text { difficulty: } M=185 \mathrm{~ms}\end{array}$ & No & $\begin{array}{c}\text { Flexor } \\
\text { digitorum }\end{array}$ \\
\hline $\begin{array}{l}\text { Miller et al. (1999) Exp } 2 \\
\text { pitch discrimination, } \\
\text { manipulation of sound } \\
\text { intensity }\end{array}$ & $\begin{array}{c}\text { Yes } \\
\text { PMT lowest } \\
\text { perceptual difficulty } \\
(M=317 \mathrm{~ms})<\text { PMT } \\
\text { highest perceptual } \\
\text { difficulty }(M=334 \\
\mathrm{ms})\end{array}$ & $\begin{array}{l}\text { No } \\
\text { MT lowest perceptual } \\
\text { difficulty: } M=160 \mathrm{~ms} ; \\
\text { MT highest perceptual } \\
\text { difficulty } M=161 \mathrm{~ms}\end{array}$ & $\begin{array}{c}\text { No } \\
\text { ER lowest perceptual } \\
\text { difficulty: } M=2.7 \% ; \\
\text { ER highest } \\
\text { perceptual difficulty: } \\
M=2.0 \%\end{array}$ & $\begin{array}{c}\text { Flexor } \\
\text { digitorum }\end{array}$ \\
\hline $\begin{array}{c}\text { Servant et al. (2016) } \\
\text { Simon task, manipulation } \\
\text { of color saturation }\end{array}$ & $\begin{array}{c}\text { Yes } \\
\text { PMT lowest } \\
\text { perceptual difficulty } \\
\text { level }(M=333 \mathrm{~ms})< \\
\text { PMT highest } \\
\text { perceptual difficulty } \\
\text { level }(M=382 \mathrm{~ms})\end{array}$ & $\begin{array}{c}\text { Yes } \\
\text { MT lowest perceptual } \\
\text { difficulty }(M=132 \mathrm{~ms}) \\
<\text { MT highest } \\
\text { perceptual difficulty ( } M \\
=134 \mathrm{~ms})\end{array}$ & $\begin{array}{c}\text { Yes } \\
\text { ER lowest perceptual } \\
\text { difficulty }(M=10.1 \%) \\
<\text { ER highest } \\
\text { perceptual difficulty } \\
(M=3.9 \%)\end{array}$ & $\begin{array}{c}\text { Flexor pollicis } \\
\text { brevis }\end{array}$ \\
\hline $\begin{array}{l}\text { Weindel et al. (2020) } \\
\text { Exp } 1 \\
\text { gabor contrast } \\
\text { discrimination }\end{array}$ & $\begin{array}{c}\text { Yes } \\
\text { PMT lowest } \\
\text { perceptual difficulty } \\
\text { ( } M=324 \mathrm{~ms})<\text { PMT } \\
\text { highest perceptual } \\
\text { difficulty }(M=424 \\
\text { ms })\end{array}$ & $\begin{array}{c}\text { Yes } \\
\text { MT lowest perceptual } \\
\text { difficulty }(M=107 \mathrm{~ms}) \\
<\text { MT highest } \\
\text { perceptual difficulty ( } M \\
=113 \mathrm{~ms})\end{array}$ & $\begin{array}{c}\text { Yes } \\
\text { ER lowest perceptual } \\
\text { difficulty }(M=45.9 \%) \\
<\text { ER highest } \\
\text { perceptual difficulty } \\
\qquad(M=2.3 \%)\end{array}$ & $\begin{array}{c}\text { Flexor pollicis } \\
\text { brevis, } \\
6 \mathrm{~N} \text { force }\end{array}$ \\
\hline
\end{tabular}




\begin{tabular}{ccccc}
\hline & Yes & Yes & Yes & \\
Weindel et al. (2020) & PMT lowest & MT lowest perceptual & ER lowest perceptual & Flexor pollicis \\
Exp 2 & perceptual difficulty & difficulty $(M=74 \mathrm{~ms})<$ & difficulty $(M=45.2 \%)$ & brevis, \\
gabor contrast & $(M=354 \mathrm{~ms})<$ PMT & MT highest perceptual & $<$ ER highest & $2 \mathrm{~N}$ force \\
discrimination & $\begin{array}{c}\text { highest perceptual } \\
\text { difficulty }(M=440\end{array}$ & difficulty $(M=78 \mathrm{~ms})$ & perceptual difficulty & \\
& $\mathrm{ms})$ & & \\
& & & \\
& & & \\
\hline
\end{tabular}

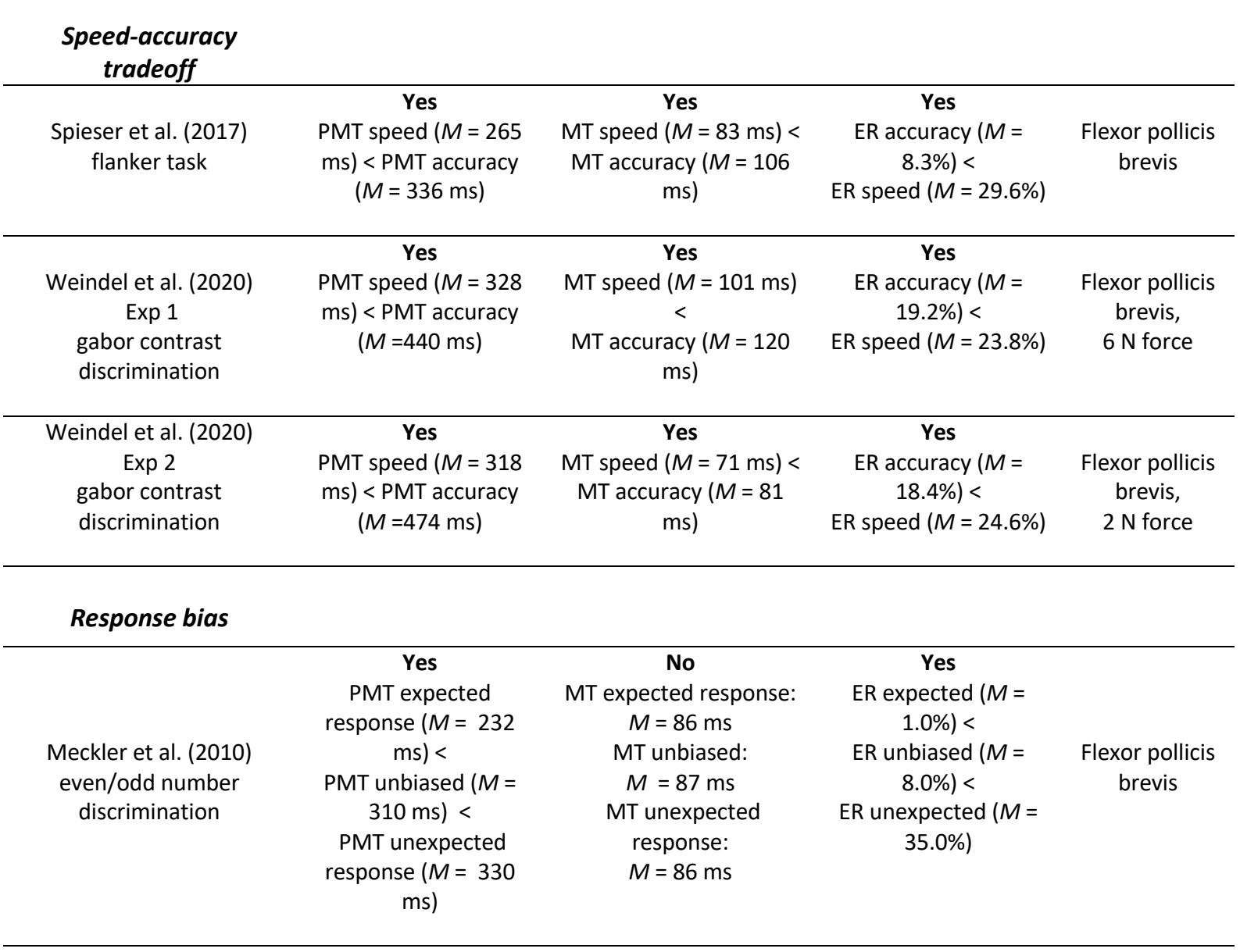

\section{Response force}

\begin{tabular}{|c|c|c|c|c|}
\hline $\begin{array}{l}\text { Romaiguère et al. (1993) } \\
\text { Simon task, manipulation } \\
\text { of light intensity }\end{array}$ & $\begin{array}{c}\text { Yes } \\
\text { PMT highest force ( } M \\
=451 \mathrm{~ms})<\text { PMT } \\
\text { lowest force }(M= \\
478 \mathrm{~ms})\end{array}$ & $\begin{array}{c}\text { Yes } \\
\text { MT lowest force }(M= \\
264 \mathrm{~ms})<M T \text { highest } \\
\text { force }(M=370 \mathrm{~ms})\end{array}$ & No & $\begin{array}{c}\text { Abductor } \\
\text { pollicis brevis } \\
\text { low (from } \\
2.45 \mathrm{~N} \text { to } \\
15.44 \mathrm{~N} \text { ) } \\
\text { versus high } \\
\text { (from } 15.46 \mathrm{~N} \\
\text { to } 63 \mathrm{~N} \text { ) force }\end{array}$ \\
\hline $\begin{array}{l}\text { Burle et al. (2002) } \\
\text { Simon task }\end{array}$ & $\begin{array}{c}\text { Yes } \\
\text { PMT highest force ( } M \\
=295 \mathrm{~ms})<\mathrm{PMT} \\
\text { lowest force }(M= \\
308 \mathrm{~ms})\end{array}$ & $\begin{array}{c}\text { Yes } \\
\text { MT lowest force }(M= \\
80 \mathrm{~ms})<\mathrm{MT} \text { highest } \\
\text { force }(M=131 \mathrm{~ms})\end{array}$ & $\begin{array}{c}\text { Yes } \\
\text { ER highest force }(M= \\
0.5 \%)< \\
\text { ER lowest force }(M= \\
1.5 \%)\end{array}$ & $\begin{array}{c}\text { Flexor pollicis } \\
\text { brevis } \\
\text { low }(0.49 \mathrm{~N}) \\
\text { versus high } \\
(9.81 \mathrm{~N}) \text { force }\end{array}$ \\
\hline
\end{tabular}

Note: Some information (e.g., effect size, muscle recorded, force required) is missing for some studies because it has not been reported in the corresponding papers. $\mathrm{N}=$ Newtons (1 Newton in earth gravity is the equivalent weight of $101.97 \mathrm{grams}$ ), $\mathrm{ms}=$ milliseconds . 


\section{Experiment}

\section{Method}

Participants. Eighteen undergraduate psychology students ( 2 men; age range: 18-32; mean age: 21.1) from the University of Franche-Comté participated in the experiment in exchange for course credits. This sample size was determined based on prior studies reporting significant perceptual effects on EMG activity (range 8-16 subjects; Romaiguère et al., 1993; Servant et al., 2016; Weindel et al., 2020). Criteria for inclusion in the study were normal or corrected-to-normal vision, no history of psychiatric or neurological disorders, and no history of injuries of the musculoskeletal system. Our experimental procedures followed the guidelines of the Research Ethics Committee of the University of Franche-Comté. Participants were not aware of the purpose of the experiment, and provided written consent to participate.

Apparatus. The experiment took place in a dimly lit room. Subjects sat on a comfortable chair at a distance of $80 \mathrm{~cm}$ from a $34.6 \times 19.4 \mathrm{~cm}$ LCD monitor (resolution: 1920 $\times$ 1080; refresh rate: $60 \mathrm{~Hz}$ ). For each subject, the height of the monitor was adjusted such that the eyes and the center of the monitor were at the same horizontal level. The experiment was programmed in Python, using components of the PsychoPy toolbox (Peirce et al., 2019), and was run on a PC computer natively running Windows 10 . Responses were communicated to the computer by means of two thermoplastic rubber hand-held buttons (Figure $3 \mathrm{~A}$ ) connected to a Black Box Toolkit module ${ }^{T M}$ designed to guarantee millisecond accuracy. The force required to push each button was approximately $8.83 \mathrm{~N}$ (data provided by the Black Box Toolkit Company ${ }^{\top \mathrm{M}}$ ). To minimize tonic motor activity and maximize comfort, subjects' hands were palm-faced down, resting on a supportive cushion placed on their laps. 
Stimuli. White dots were presented within a virtual $12.6^{\circ}$ circular aperture centered on a $24.8^{\circ} \times 13.9^{\circ}$ black field. Each dot was a $4 \times 4$ pixel $\left(.05^{\circ}\right.$ square $)$, moving at a speed of $8^{\circ} \%$ s. Dot density was fixed at 16.7 dots $\operatorname{deg}^{-2} \mathrm{~s}^{-1}$. Random dot motion was controlled by a white noise algorithm (Pilly \& Seitz, 2009): from each frame to the next, a proportion $p$ of dots was randomly selected to move in the signal direction (leftward versus rightward), and the remaining dots were plotted in random locations. We refer to $p$ as motion coherence, which varied according to 6 levels $(0, .05, .08, .12, .2, .4)$.

Procedure. Participants were instructed to identify motion direction (leftward versus rightward) and press the corresponding button with their left or right hand. They were further instructed to respond as quickly and accurately as possible. Each trial was determined by a factorial combination of motion direction (leftward versus rightward) and motion coherence $(0, .05, .08, .12, .2, .4)$. All types of trials occurred equally often, and were presented in a random order. Each trial started with the presentation of the random dot motion stimulus, which remained on the screen until the participant responded. A RT deadline was set to $5 \mathrm{~s}$. If participants failed to respond by then, "Too slow! Please respond faster" was displayed in red on the screen for $1.5 \mathrm{~s}$. The interval between the response to the stimulus and the next trial was 1.5 s. Participants first completed 24 practice trials (each of the 12 possible stimuli presented 2 times in a random manner) to ensure they understood the task. Each practice trial was followed by a feedback on performance ("Correct response" displayed in green after a correct response; "Incorrect response" displayed in red after an incorrect response). Participants then worked through 12 blocks of 96 trials each, with a short break between blocks. Practice trials were discarded from analyses.

EMG recordings and signal processing. The EMG activity of the response agonist (flexor pollicis brevis) of each hand was recorded by means of two $\mathrm{Ag} / \mathrm{Cl}$ active electrodes 
connected to a Biosemi ActiveTwo Mk2 biopotential measurement system (sampling rate = $1024 \mathrm{~Hz}$ ). Electrodes were fixed $1 \mathrm{~cm}$ apart on the skin of the thenar eminence (Figure 3A). After data acquisition, EMG signals were processed in Python, using components of the MEG+EEG analysis \& visualization (MNE) toolbox (Gramfort et al., 2013) and signal processing routines that will soon be released with an open-source license (Spieser \& Burle, in preparation). For each subject, signals were bipolar re-referenced, high-pass filtered using a $10 \mathrm{~Hz}$ cut-off ( $3^{\text {rd }}$ order Butterworth filter), and epoched -0.5 to $5 \mathrm{~s}$ relative to stimulus onset. For each epoch, EMG burst onsets were detected using a three-step procedure. We first performed a detection of EMG activity by applying a threshold on signal variance. Specifically, we computed the mean $M_{b}$ and standard deviation $S D_{b}$ of the rectified signal in the $0.5 \mathrm{~s}$ baseline period. Periods of EMG activity corresponded to time windows in which rectified signal amplitudes exceeded the following criterion:

$$
\text { criterion }=M_{b}+3.5 \times S D_{b}
$$

Due to the oscillatory nature of EMG activity, we further merged consecutive time windows separated by less than $0.025 \mathrm{~s}$. Although this method is widely used to detect EMG onsets (defined as the first voltage sample above the criterion), it is sensitive to fluctuations in the signal-to-noise ratio, the presence of artifacts during the baseline period, and involves an arbitrary choice (the number of standard deviations) for setting the threshold. To attenuate these issues, we next defined new time windows bounded halfway between active EMG windows identified by the previous method. We then applied the integrated profile method to each window (Liu \& Liu, 2016; Santello \& McDonagh, 1998), as recommended by Spieser and Burle (in preparation). The method first requires to compute the integrated profile $I P(t)$, defined as the cumulative sum of rectified voltage datapoints. A reference line $R(t)$, defined as the cumulative sum of an equally distributed signal, is then computed as follows: 


$$
R(t)=I P(M) \times \frac{t}{M}
$$

where $M$ corresponds to the length of the time window. Taking the minimum of the difference $I P(t)-R(t)$ gives the EMG onset (for an illustration, see Supplementary Figure S4). Note that the integrated profile method can detect only one EMG onset per time window, so it must be preceded by the threshold method to identify windows of active EMG activity in the trial. As a final step, each trial was inspected visually by the experimenter, and erroneous EMG onsets were manually corrected. All triggers relative to stimulus and response identification were removed, such that the experimenter was not aware of the experimental condition the epoch belonged to. For each trial, RT was then divided into a PMT (from stimulus onset to EMG onset) and a MT (from EMG onset to the response; Figure 1B). When the trial contained partial EMG bursts, PMT and MT were defined relative to the EMG onset of the response. EMG onsets could sometimes not be detected due to high tonic motor activity or electrical noise $\mathbf{7 . 5 \%}$ of trials on average; range $0.2-24 \%)$, and these trials were discarded from all analyses.

Data analyses. Anticipations (RTs $<150 \mathrm{~ms} ; 0.13 \%$ ) and trials in which participants failed to respond before the $5 \mathrm{~s}$ deadline (0.14\%) were discarded from all analyses. Chronometric data and accuracy data ${ }^{2}$ were analyzed by means of repeated-measures ANOVAs with motion coherence as factor. The sphericity assumption was evaluated by Mauchly's test (Mauchly, 1940). When sphericity was violated, degrees of freedom were corrected according to the procedure developed by Greenhouse and Geisser (1959). We also report the respective Bayesian analyses, i.e the Bayes factor (BF) in favor of the model incorporating the effect of motion coherence (alternative hypothesis $\mathrm{H} 1$ ) relative to the null model without the effect (null hypothesis HO). Our interpretation of the BF was based on

\footnotetext{
${ }^{2}$ When motion coherence was null, the expected correct response (left versus right) was chosen randomly.
} 
Jeffreys (1961): $\mathrm{BF}_{10}>30$ : very strong evidence for the effect, $30>\mathrm{BF}_{10}>10=$ strong evidence, $10>\mathrm{BF}_{10}>3=$ moderate evidence, $3>\mathrm{BF}_{10}>1=$ weak evidence. We also report the error percentage (due to the Markov chain Monte Carlo algorithm) associated with each BF to inform the reader about the numerical stability of the results, as recommended by van Doorn et al. (2020). Although our chronometric analyses focus on correct trials, the results were unchanged when considering all trials.

\section{Results}

RT and accuracy data. An ANOVA on mean RT data in correct trials with motion coherence as within-subject factor showed a significant effect of motion coherence $\left(F(1.38,23.39)=43.16, M S E=0.10, p<.001, \eta^{2}=.72\right)$ and very strong evidence for this effect $\left(\mathrm{BF}_{10}=4.48 \times 10^{18}\right.$, error $\left.\%=0.45\right)$. Figure $3 \mathrm{~B}$ shows that mean $\mathrm{RT}$ increases as motion coherence decreases (amplitude of the effect: $M=726 \mathrm{~ms}$; see Table 2). An ANOVA on accuracy data with motion coherence as within-subject factor showed a significant effect of motion coherence $\left(F(1.70,28.91)=151.35, M S E=0.011, p<.001, \eta^{2}=.90\right)$ and very strong evidence for this effect $\left(\mathrm{BF}_{10}=1.77 \times 10^{38}\right.$, error $\left.\%=1.06\right)$. Figure $3 \mathrm{~B}$ shows that accuracy decreases as motion coherence decreases.

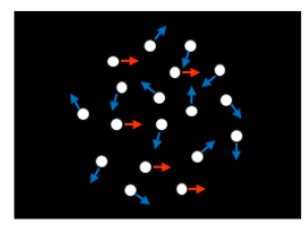

A

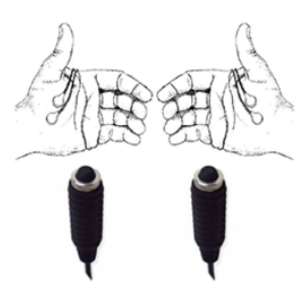

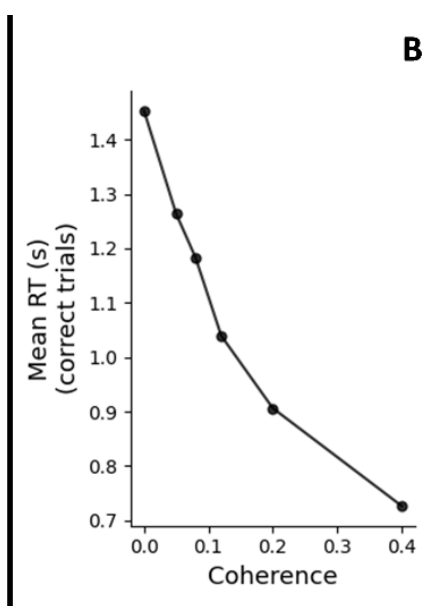

B

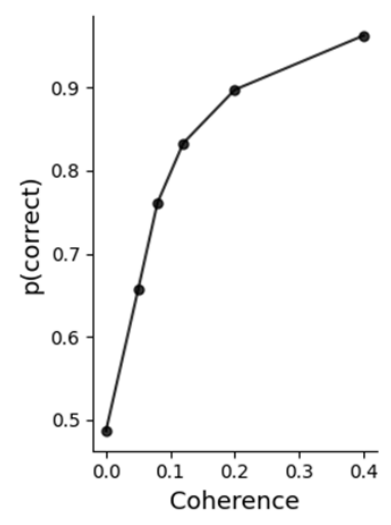


Figure 3. Random dot motion task and associated behavioral performance. A) In each trial, subjects were presented with an array of dots moving in random directions (blue arrows), with a proportion moving coherently leftward or rightward (red arrows). This proportion, termed motion coherence, varied randomly from trial-to-trial according to 1 of 6 levels $(0, .05, .08$, $.12, .2, .4)$. Subjects had to discriminate the coherent motion direction, and press the corresponding button with their left or right thumb. The EMG activity of the response agonist of each hand (flexor pollicis brevis) was recorded by means of two electrodes fixed $1 \mathrm{~cm}$ apart on the skin of the thenar eminence. B) Subjects showed the typical behavioral performance in this task, with slower and less accurate responses as motion coherence decreases.

Table 2. Chronometric and accuracy data averaged across subjects. Chronometric data are computed from correct trials only.

\begin{tabular}{ccccccc}
\hline & \multicolumn{7}{c}{ Motion coherence levels } \\
\cline { 2 - 7 } & 0 & .05 & .08 & .12 & .2 & .4 \\
\hline Mean response time (s) & 1.453 & 1.263 & 1.183 & 1.039 & 0.906 & 0.726 \\
Mean premotor time (s) & 1.269 & 1.085 & 1.010 & 0.872 & 0.746 & 0.577 \\
Mean motor time (s) & 0.184 & 0.178 & 0.173 & 0.167 & 0.160 & 0.149 \\
Accuracy & 0.49 & 0.66 & 0.76 & 0.83 & 0.90 & 0.96 \\
\hline
\end{tabular}

Prediction 1. According to DTDM, both mean PMT and mean MT should increase as motion coherence decreases (Figure 2C). ANOVAs on mean PMT and mean MT in correct trials with motion coherence as within-subjects factor revealed significant effects of motion coherence on PMT $\left(F(1.37,23.32)=40.03, M S E=0.10, p<.001, \eta^{2}=.70\right)$ and on MT $\left(F(2.24,38.06)=25.86, M S E=0.0002, p<.001, \eta^{2}=.60\right)$, and very strong evidence for these effects $\left(\mathrm{BF}_{10}=5.18 \times 10^{17}\right.$ and $\mathrm{BF}_{10}=4.53 \times 10^{12}$ respectively, error $\%=0.41$ and 0.41 respectively). Figure $4 \mathrm{~A}$ shows that both mean PMT and mean $\mathrm{MT}$ increase as motion coherence decreases (amplitude of the effect on PMT: $M=692 \mathrm{~ms}$; amplitude of the effect on MT: $M=35$ ms; see Table 2), consistent with DTDM's prediction. 

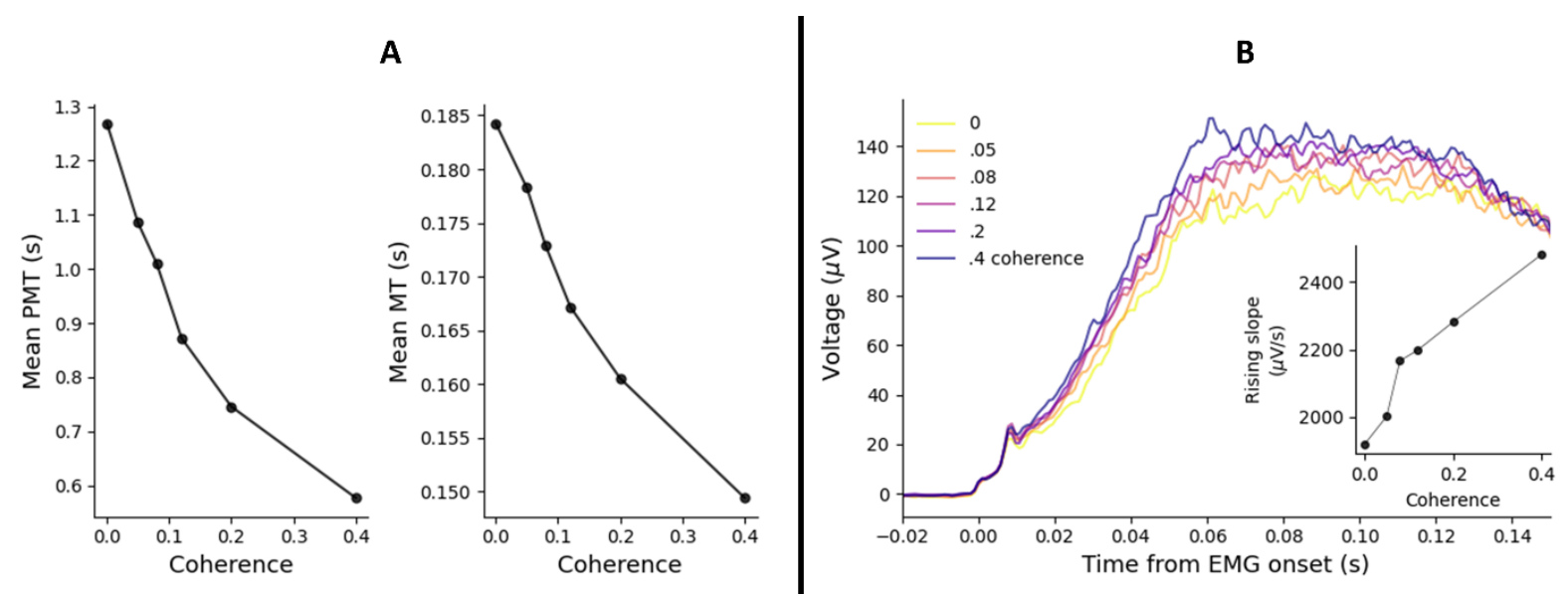

Figure 4. A) Mean PMT and mean MT in correct trials as a function of motion coherence (black points). Red crosses are predictions from the best-fitting chronometric function of the standard diffusion model. B) Rectified EMG bursts for each motion coherence condition in correct trials averaged across subjects. Signals are time-locked to EMG onset, and baselinecorrected with respect to the $100 \mathrm{~ms}$ window prior to the locking event. The inset shows the rising slope of EMG bursts (estimated by linear regression in the $60 \mathrm{~ms}$ window after EMG onset) averaged across subjects for each coherence level.

Prediction 2. DTDM predicts that the rising slope of rectified and averaged EMG bursts for each motion coherence condition reflects the drift rate, and should thus decrease as motion coherence decreases. To test this hypothesis, we first rectified EMG signals for each subject and segmented correct trials -.1 to $.5 \mathrm{~s}$ relative to EMG onset. The signals were then baseline corrected relative to the 100 ms window before EMG onset, and averaged for each motion coherence condition separately. We then estimated the rising slope of averaged EMG bursts using linear regression in the $60 \mathrm{~ms}$ window after EMG onset, and submitted these slopes to an ANOVA with motion coherence as within-subjects factor. This analysis revealed a significant effect of coherence $\left(F(2.83,48.10)=11.16, M S E=115062, p<.001, \eta^{2}=.40\right)$ and very strong evidence for this effect $\left(\mathrm{BF}_{10}=3.96 \times 10^{5}\right.$, error $\left.\%=0.39\right)$. As illustrated in Figure $4 \mathrm{~B}$, the rising slope of averaged EMG bursts decreased as motion coherence decreased, consistent with DTDM's prediction. 
Prediction 3. DTDM predicts partial EMG bursts during PMT when the decision variable oscillates around the EMG threshold (due to noise in the evidence accumulation process). The proportion of partial EMG bursts should increase as motion coherence decreases, because there is less evidence (motion energy) to drive the decision variable to the response threshold. For the same reason, the mean latency of the first partial EMG burst should increase as motion coherence decreases.
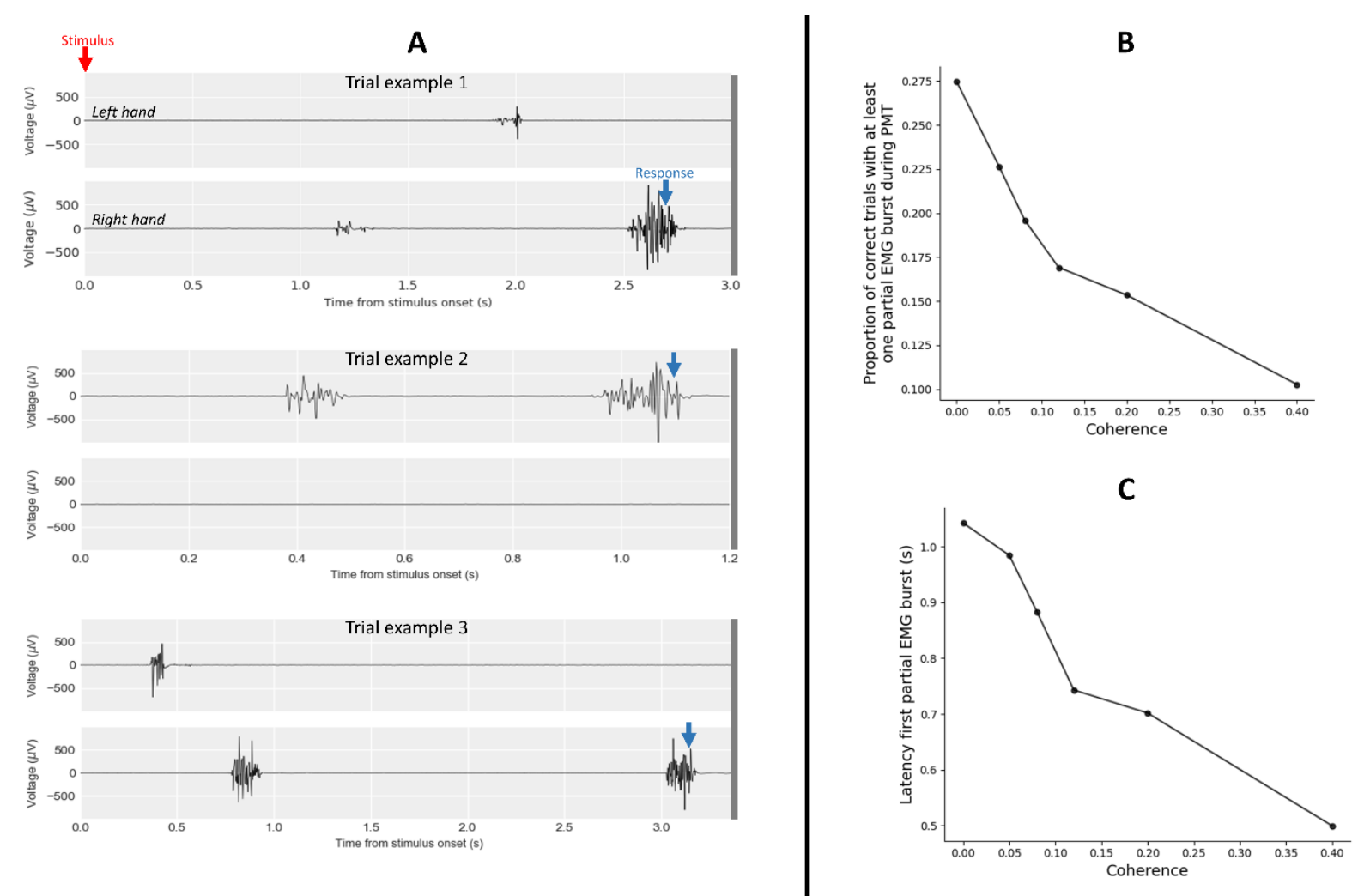

Figure 5. A) Examples of trials featuring at least one partial EMG burst during PMT. B) The proportion of such trials increases as motion coherence decreases. C) The latency of the first partial EMG burst relative to stimulus onset increases as motion coherence decreases.

Consistent with previous work (Gajdos et al., 2019; Weindel et al., 2020), we sometimes observed trials featuring one or more partial EMG burst during PMT. Examples of such trials are provided in Figure 5A. An ANOVA on the proportion of correct trials featuring at least 1 partial EMG burst during PMT with motion coherence as within-subjects factor showed a significant effect of coherence $\left(F(2.54,43.13)=25.12, M S E=0.005, p<.001, \eta^{2}=.60\right)$, 
and very strong evidence for this effect $\left(\mathrm{BF}_{10}=2.39 \times 10^{13}\right.$, error $\left.\%=0.44\right)$. Figure $5 \mathrm{~B}$ shows that the proportion of correct trials featuring at least 1 partial EMG burst during PMT increases as motion coherence decreases, consistent with DTDM prediction. An ANOVA on the mean latency of the first partial EMG burst during PMT with motion coherence as within-subjects factor showed a significant effect of coherence $(F(1.72,29.29)=27.47, M S E=0.077, p<.001$, $\left.\eta^{2}=.62\right)$, and very strong evidence for this effect $\left(\mathrm{BF}_{10}=2.37 \times 10^{13}\right.$, error $\left.\%=0.37\right)$. Figure $5 \mathrm{C}$ shows that the mean latency of the first partial EMG burst during PMT increases as motion coherence decreases, consistent with DTDM's prediction. Note that this analysis rules out an alternative explanation of partial EMG bursts in terms of motor noise, because a motor noise account predicts no effect of coherence on the mean latency of the first partial EMG burst (contrary to DTDM).

Prediction 4. DTDM predicts that the distributions of PMT and MT should exhibit a similar right-skewed shape for each coherence level. Figure 6 shows histograms of PMT (row 1) and MT (row 2) in correct trials across motion coherence levels (columns). Histograms were constructed by averaging PMT and MT deciles across subjects (Ratcliff, 1979). The width of each bar is determined by the latency difference between adjacent quantiles, and the height of each bar corresponds to the reciprocal of the width. Both dependent variables seem to exhibit a similar right-skewed shape. To assess whether it is the case, we constructed PMTMT quantile-quantile (Q-Q) plots. Figure 6 (row 3) shows that the $Q-Q$ plot averaged across subjects is well described by a linear fit, suggesting that PMT and MT have a similar shape, consistent with DTDM's prediction. Additional support for this hypothesis comes from the very high and positive Pearson's correlation coefficient between PMT and MT deciles at the individual level (Table 3). 
Table 3. Pearson's correlation coefficient between PMT and MT deciles in correct trials for each motion coherence level.

\begin{tabular}{ccccccc}
\hline & \multicolumn{5}{c}{ Coherence level } \\
\cline { 2 - 7 } & 0 & .06 & .08 & .12 & 2 & .4 \\
\hline $\begin{array}{c}\text { Pearson's } r \\
\text { averaged } \\
\text { across subjects }\end{array}$ & .978 & .985 & .985 & .980 & .987 & .990 \\
\hline $\begin{array}{c}\text { Range (min- } \\
\text { max) }\end{array}$ & $.890-.993$ & $.950-.998$ & $.956-.997$ & $.920-.999$ & $.949-.999$ & $.978-.997$ \\
\hline $\begin{array}{c}\text { Standard } \\
\text { deviation }\end{array}$ & .024 & .012 & .011 & .019 & .013 & .005 \\
\hline
\end{tabular}

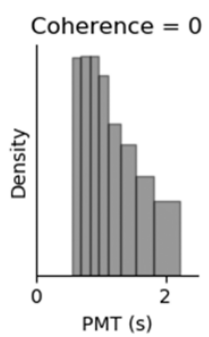

.05
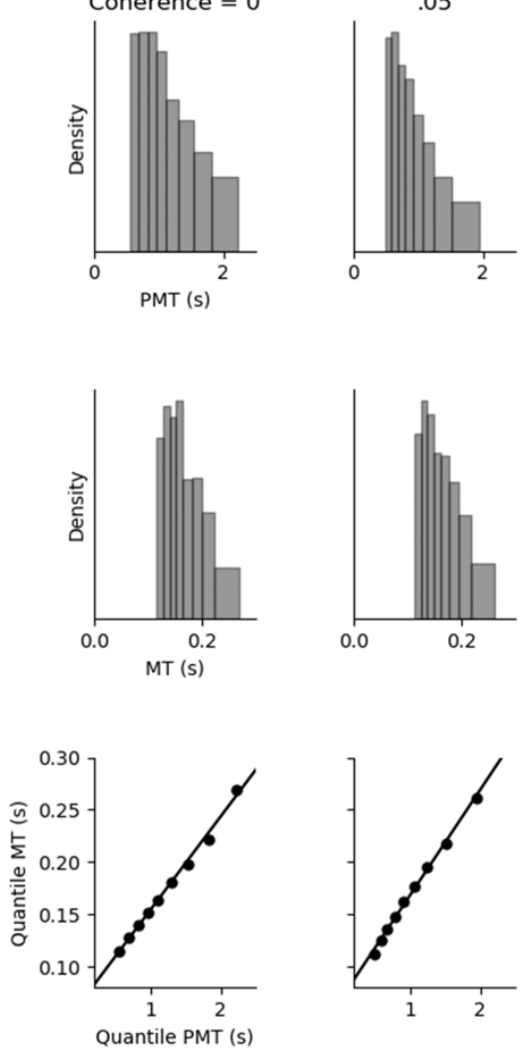

PMT histograms
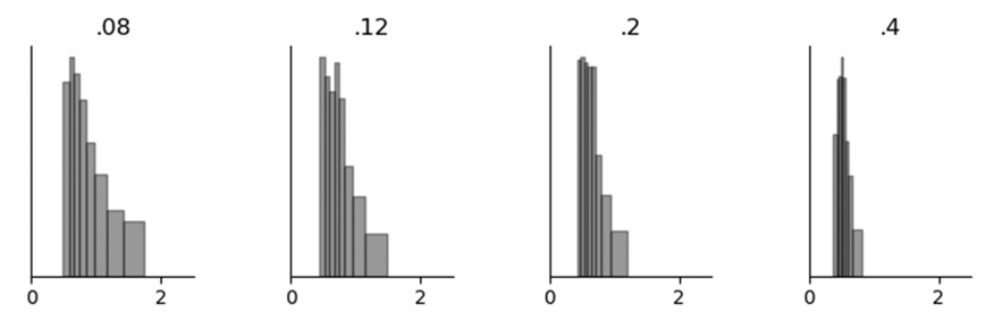

MT histograms
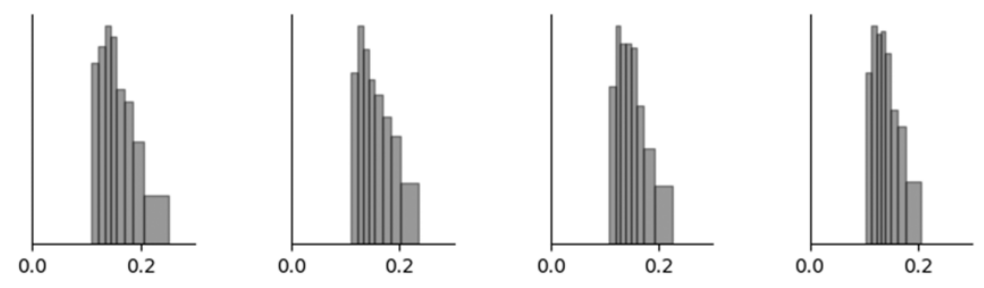

Q-Q plots
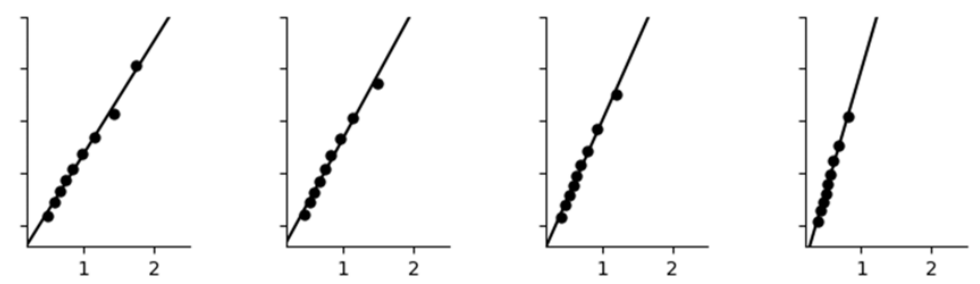

Figure 6. Histograms of PMT (row 1 ) and MT (row 2) in correct trials across motion coherence levels (columns) averaged across subjects. Also shown is a quantile-quantile (Q-Q) plot of PMT versus MT deciles for each coherence level averaged across subjects (row 3), and the corresponding regression line. See text for details. 
Prediction 5. Without between-trial variability in drift rate, DTDM predicts a null between-trial correlation between PMT and MT. However, a modest amount of between-trial variability in drift rate, as classically assumed in the field, would generate a small positive correlation between PMT and MT. To test this prediction, we computed the between-trial Pearson correlation coefficient between PMT and MT for each subject and motion coherence level. These correlations are illustrated in Figure 7. For each coherence level, the averaged coefficient is small and positive, consistent with our prediction. Interestingly, the averaged coefficient is slightly smaller for the highest coherence level compared to lower levels. This pattern may reflect an increase in between-trial variability in drift rate as coherence decreases, which may stem from the increased amount of noise in the stimuli.
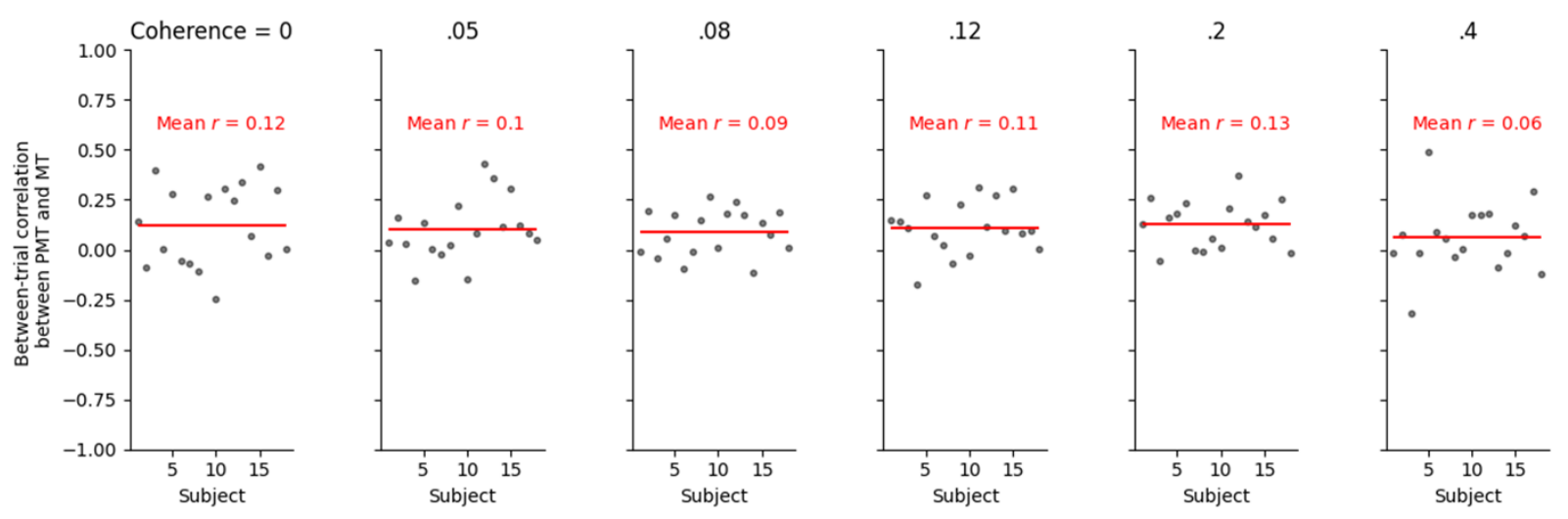

Figure 7. Between-trial Pearson correlation coefficient between MT and PMT for each motion coherence level in correct trials. Black points show data from individual subjects. Each red line shows the coefficient averaged across subjects.

\section{Discussion}

Our empirical data provides strong evidence for each of the five qualitative benchmark predictions derived from DTDM. Nevertheless, a complete test of the model requires a quantitative fit to both behavioral and EMG data. We also fit the standard DDM to behavioral 
data, in order to evaluate the relationship between the best-fitting parameters from the two models.

Fits of the DDM to behavioral data usually incorporate between-trial variability in drift rate $s v$, starting point $s z$, and nondecision time $s T e r$. However, parameter recovery studies have shown that $s v$ and $s z$ are poorly recovered, and tradeoff with the main model parameters (Boehm et al., 2018; Lerche \& Voss, 2017; Ratcliff \& Tuerlinckx, 2002). Because these tradeoffs could bias the comparison between DTDM and DDM, we decided to fix sv and $s z$ to zero in our modeling. We also removed any source of variability in nondecision time components to ensure that good fits to PMT and MT data are not caused by these additional parameters. The drift rate was the only parameter allowed to vary between coherence levels. Because left and right responses were equiprobable in our random dot motion task, the starting point of evidence accumulation was fixed to zero (halfway between thresholds). Consequently, the DDM had eight free parameters: one drift rate $v$ for each coherence level, upper decision threshold $a$, and mean nondecision time Ter. DTDM had 10 free parameters: one drift rate $v$ for each coherence level, upper EMG threshold $m$, upper response threshold $r$, mean residual latency Te added to predicted PMT (comprising at least the sensory encoding time and the corticomuscular delay), and mean residual latency $\operatorname{Tr}$ added to predicted MT (comprising the electromechanical delay).

The next section is structured as follows. First, we simultaneously fit the DDM to the RT distributions of correct and incorrect responses and to accuracy data using a standard quantile-based method (Ratcliff \& Smith, 2004; Smith \& Lilburn, 2020; Smith \& Ratcliff, 2009). Following previous work (Ratcliff \& McKoon, 2008), we anticipated slower errors than correct responses in our data that the model should miss because we fixed $s v$ to 0 . Apart from this 
minor misfit, we predicted that the standard DDM should provide a good account of the data. Next, we fit DTDM to the joint distributions of PMT and MT in correct and incorrect trials and to accuracy data using the same quantile-based method. Based on the theoretical foundations built from our previous qualitative assessments of the model, we predicted a good quantitative fit to data. Finally, we compare DDM and DTDM best-fitting parameters using between-subjects correlations and linear regressions.

\section{Modeling}

\section{Method}

DDM fit procedure. Although closed-form expressions exist for the DDM (without between-trial variability in any of the model parameters), we simulated the model using the method and framework of Evans (Evans, 2019) to allow for a direct comparison with DTDM. The time-step $d t$ was fixed at $0.001 \mathrm{~s}$ and the diffusion coefficient was fixed at 0.1 . The model was coded in C, and the fit procedure was coded in Python.

The model was fit to each individual behavioral dataset by minimizing the likelihoodratio chi-square statistic $G^{2}$ (Ratcliff \& Smith, 2004; Servant et al., 2019; Smith \& Lilburn, 2020; Smith \& Ratcliff, 2009):

$$
G^{2}=2 \sum_{i=1}^{6} \sum_{j=1}^{2} \sum_{k=1}^{6} n_{i j k} \ln \left(\frac{n_{i j k}}{\frac{\text { pred_n } n_{i j k} N_{i}}{\text { simul_ } N_{i}}}\right)
$$

The outer summation over $i$ extends over the six motion coherence conditions, and the summation over $j$ extends over the two trial types (correct vs incorrect). The summation over 
$k$ extends over the six bins bounded by RT quantiles $(.1, .3, .5, .7 \text {, and } .9)^{3}$. The variables $n_{i j k}$ and $p r e d \_n_{i j k}$ refer to the observed and predicted number of trials in coherence condition $i$, trial type $j$, and RT bin $k$. Finally, the variables $N_{i}$ and simul_N $N_{i}$ refer to the observed and simulated number of trials in coherence condition $i$. The $G^{2}$ statistic thus characterizes the goodness-of-fit of the model to the correct and error RT distributions and to the correct and error choice probabilities simultaneously.

The $G^{2}$ statistic was minimized using a differential evolution optimizer (Storn \& Price, 1997), and 20,000 simulated trials per coherence condition. Results were polished with a Simplex optimizer (Nelder \& Mead, 1965), using 100,000 simulated trials per coherence condition. Codes were run on the advanced computing center from the University of FrancheComté.

DTDM fit procedure. DTDM was simulated using the exact same method as for the DDM. An implementation of the model in C language is provided in Appendix $A$ of Supplemental Materials. In each trial, predicted PMT and MT are defined as follows:

$$
\begin{gathered}
\text { predicted } P M T=T e+x \\
\text { predicted } M T=T r+y
\end{gathered}
$$

The variable $x$ corresponds to the latency between accumulation onset and the last crossing of an EMG bound before the response. The variable $y$ corresponds to the latency between the last crossing of an EMG bound and the corresponding response bound. Since observed PMT and MT were defined with respect to the last EMG burst giving the response, our computational definition is fully consistent with observed data.

\footnotetext{
${ }^{3}$ If subjects made a number of errors comprised between 5 and 10 in a given condition, a median split was used to form two bins. If there were fewer than 5 errors, error RTs for the condition were excluded from the $G^{2}$ computation.
} 
The model was fit to each individual dataset by minimizing the likelihood-ratio chisquare statistic $G^{2}$ :

$$
G^{2}=2 \sum_{i=1}^{6} \sum_{j=1}^{2} \sum_{k=1}^{6} \sum_{l=1}^{6} n_{i j k l} \ln \left(\frac{n_{i j k l}}{\frac{\text { pred_n } n_{i j k l} N_{i}}{\text { simul_Ni }}}\right)
$$

The outer summation over $i$ extends over the six motion coherence conditions, and the summation over $j$ extends over the two trial types (correct vs incorrect). The summation over $k$ extends over the six bins bounded by PMT quantiles (.1, .3, .5, .7, and .9), and the summation over $l$ extends over the six bins bounded by MT quantiles $(.1, .3, .5, .7$, and .9). The variables $n_{i j k l}$ and pred_n$n_{i j k l}$ refer to the observed and predicted number of trials in coherence condition $i$, trial type $j$, PMT bin $k$, and $\mathrm{MT}$ bin $l$. The $G^{2}$ statistic thus characterizes the goodness-of-fit of the model to the joint distribution of PMT and MT in correct and error trials and to the correct and error choice probabilities simultaneously. It was minimized using the exact same method as for the DDM.

\section{Results}

The observed behavioral data and best-fitting predictions from the DDM averaged across subjects are shown in Figure $8 \mathrm{~A}$ as quantile probability functions (QPFs). QPFs are constructed by plotting RT quantiles (y-axis) of the distributions of correct and incorrect responses for each experimental condition against the corresponding response type proportion (x-axis). Individual fits are shown in Supplementary Figure S5. The model provides a good account of the data. As anticipated, the only apparent misfit concerns the relative speed of correct and error responses, due to the lack of between-trial variability in drift rate.

DTDM fits to PMT and MT distributions in correct and error trials and to accuracy data averaged across subjects are shown in Figure 8B as QPFs. Also shown are RT predictions 
computed from best-fitting parameters. The model provides a good account of the data, and does not show significant misfits beyond the relative speed of correct and error responses, even at the individual level (Supplementary Figure S6). RT predictions are virtually similar to DDM predictions, which allows for a straightforward parametric comparison between the two models.

A: DDM fits

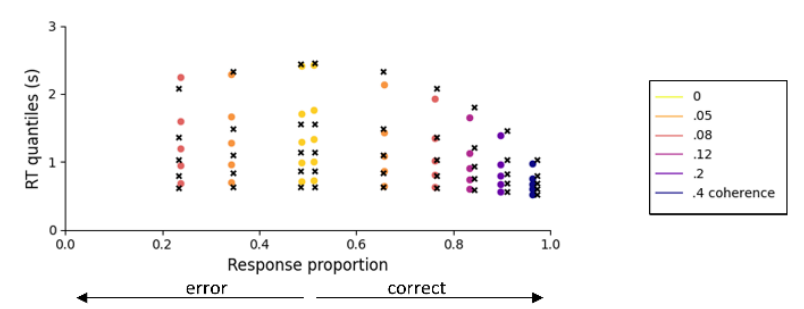

B: DTDM fits

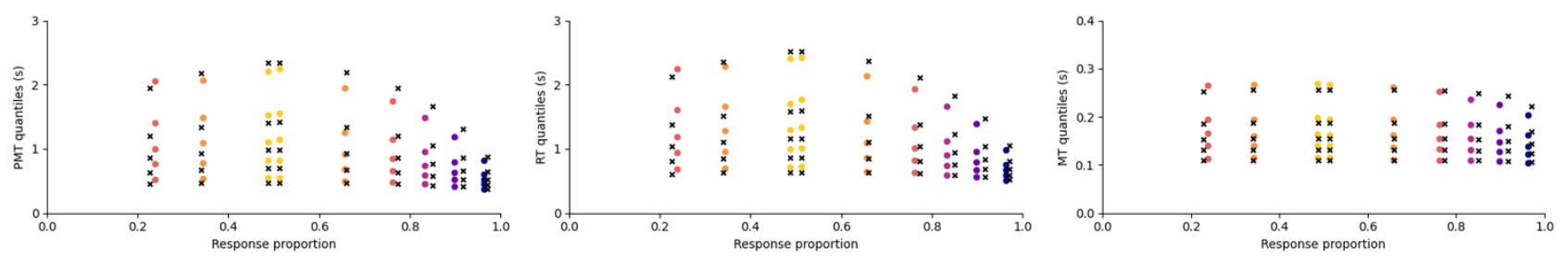

Figure 8. Observed and predicted quantile probability functions from the DDM $(A)$ and from DTDM (B) averaged across subjects. Observed data are presented as colored circles and model predictions as black $\mathrm{x}$ 's.

Best-fitting DDM and DTDM parameters averaged across subject are shown in Table 4, and scatterplots of individual best-fitting DDM versus DTDM values for each parameter are shown in Figure 9. For motion coherence levels higher than 0, drift rates from DDM and DTDM are very similar, as indicated by comparable averaged best-fitting values, high betweensubjects correlation coefficients (all Pearson's $r s \geq .95$ ), and regression lines close to the $y=$ $x$ identity line. The correlation is considerably weaker for the $0 \%$ coherence level, presumably because individual values are gathered in a very small interval around 0 due to the lack of motion energy. The predicted mean nondecision time from DTDM, defined as the sum of 
parameters $T e$ and $T r$, is virtually identical to the mean nondecision time Ter of the DDM (Pearson's $r=.97 ;$ regression line: $T e+T r=0.98 T e r)$. In addition, our model fits suggest a correspondence between the upper decision threshold $a$ from the DDM and the upper response threshold $r$ from DTDM (Pearson' $r=.97$; regression line: $r=0.93 a+0.008$ ). The upper EMG threshold $m$ from DTDM is about twice smaller on average than the upper decision threshold $a$ from the DDM (see Table 4), though the two variables strongly correlate (Pearson's $r=.86)$. Finally, we computed the correlation between the upper EMG threshold $m$ and the upper response threshold $r$. Recall that the distance between EMG and response thresholds within DTDM is only determined by the force required to respond, so the two types of thresholds are expected to correlate strongly. Consistent with this hypothesis, we found a high correlation coefficient between $m$ and $r$ parameters, Pearson's $r=.84$.

Table 4. Best-fitting parameters from DDM and DTDM models averaged across subjects.

\begin{tabular}{|c|c|c|c|c|c|c|c|c|c|c|c|c|}
\hline & \multicolumn{6}{|c|}{ drift rate $v$} & \multicolumn{3}{|c|}{ thresholds } & \multicolumn{3}{|c|}{ residual latencies (s) } \\
\hline & 0 & .05 & .08 & .12 & .20 & .40 & $a$ & $m$ & $r$ & Ter & $T e$ & $T r$ \\
\hline DDM & -0.003 & 0.034 & 0.069 & 0.111 & 0.177 & 0.318 & 0.096 & & & 0.375 & & \\
\hline DTDM & -0.001 & 0.035 & 0.069 & 0.111 & 0.177 & 0.310 & & 0.048 & 0.098 & & 0.298 & 0.070 \\
\hline
\end{tabular}

Note. DDM parameters: drift rate $v$, upper decision threshold $a$, mean residual time Ter; DTDM parameters: drift rate $v$, upper EMG threshold $m$, upper response threshold $r$, mean residual latency $T e$ added to predicted PMT (comprising at least the sensory encoding time and the corticomuscular delay), and mean residual latency $T r$ added to predicted MT (comprising the electromechanical delay). 

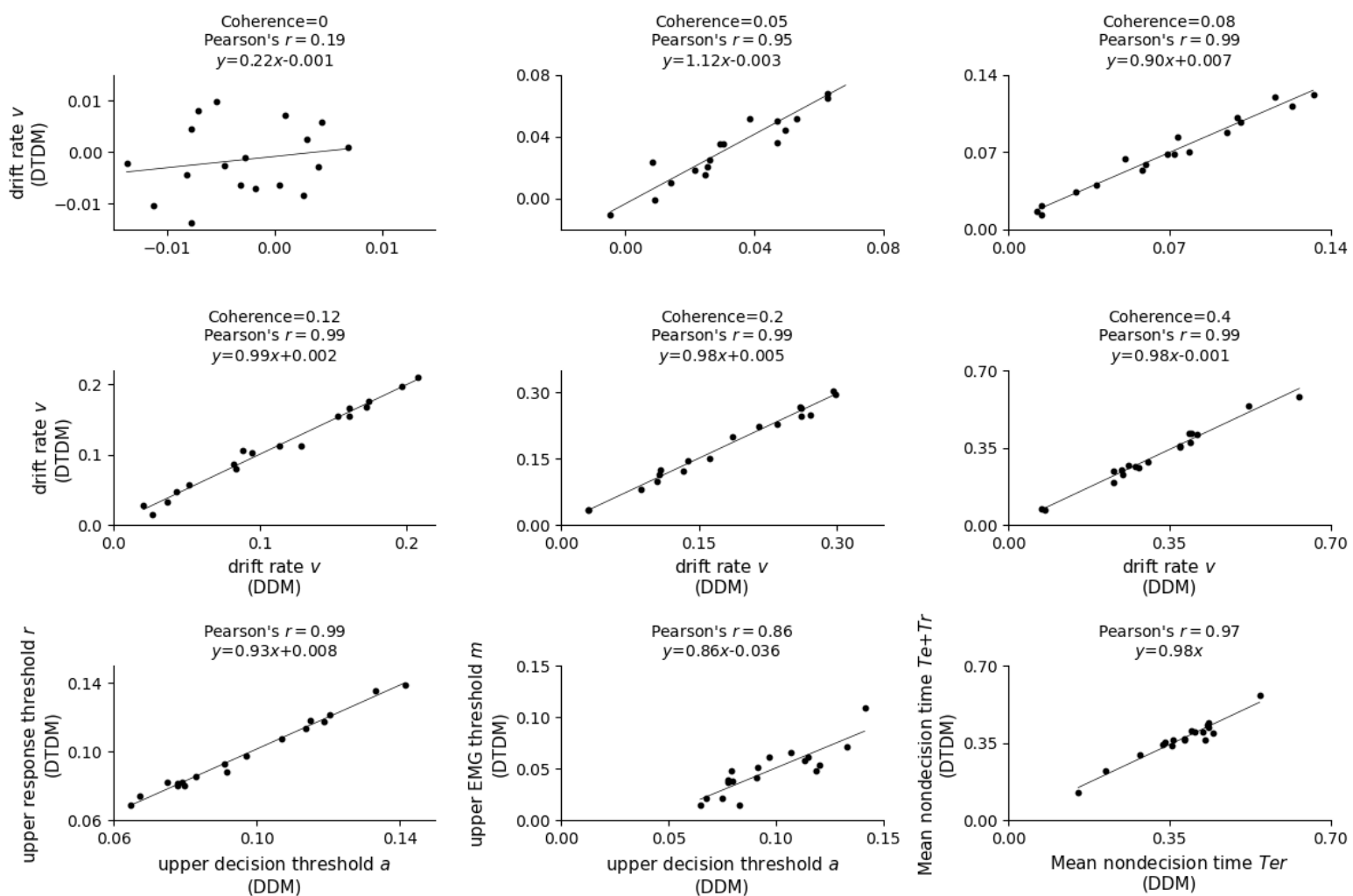

Figure 9. Scatterplots of individual best-fitting DTDM ( $y$-axis) versus DDM ( $x$-axis) values for each parameter. Also shown is the corresponding regression line.

\section{Discussion}

Our modeling provides strong quantitative evidence for DTDM, and complements previous qualitative evaluations of the model. The model accounts for the distributions of PMT and MT in correct and error trials and for the correct and error choice probabilities simultaneously, with no systematic misfit beyond the relative speed of correct and error responses, which was expected because we did not incorporate between-variability in any of the model parameters. Residual motor latencies $\operatorname{Tr}$ range 32-103 ms with a mean of $70 \mathrm{ms,}$ which fits nicely with previous empirical estimates of the electromechanical delay (range 30100 ms; Cavanagh \& Komi, 1979). 
Though DTDM was fit to the joint distribution of PMT and MT, RT predictions of the model (computed from best-fitting parameters) match those from the standard DDM. A comparison of best-fitting parameters from the two models showed that drift rates and residual latencies also match. Importantly, this analysis revealed a correspondence between decision thresholds from the DDM and response thresholds from DTDM. Thus, thresholds and residual components from the DDM appear to have been misinterpreted in previous applications of the model (e.g., Ratcliff, 1978; Ratcliff \& McKoon, 2008; Ratcliff \& Rouder, 1998; Ratcliff \& Smith, 2004; Voss et al., 2004). DTDM clarifies this issue by providing an explanation of the computations involved in the motor execution stage, and shed new light onto conflicting findings regarding selective influence, as detailed in the General Discussion.

Note that DTDM reduces to DDM when $|m|=|r|$. Because of the way muscles work, this difference must always be greater than 0 . Thus, DDM may always be false but the difference between DTDM and DDM may be too small to detect. This means that DDM fits are close to being valid when response force is low, as in many experiments. Researchers should nevertheless be careful when attributing effects of experimental factors onto the mean nondecision time parameter Ter of the DDM. DTDM assumes that the residual latency at the motor execution stage $\operatorname{Tr}$ comprises the electromechanical delay. Given the strong contribution of mechanical factors, the electromechanical delay is unlikely to be modulated by cognitive factors, though this hypothesis should be carefully evaluated in future work. In addition, the electromechanical delay is relatively short, so large modulations of Ter are likely to be caused by processes prior to the decision process, such as sensory encoding, attention filtering, and short-term memory. 


\section{General discussion}

DTDM enriches the standard diffusion model framework by providing a theory of motor execution. Specifically, the model assumes that EMG activity is determined by the same evolving decision variable that drives RT. This assumption is formalized by two sets of thresholds operating on the decision variable: EMG thresholds $( \pm m)$ and response thresholds $( \pm r)$. DTDM has proven to capture a broad range of EMG and behavioral phenomena in conflict tasks (Servant et al., 2015). The aim of the present work was to determine whether DTDM can predict EMG and behavioral data in standard perceptual decision-making tasks as well. We first derived five general predictions from the model that we submitted to an empirical test:

(1) Mean PMT and mean MT should monotonically increase as motion coherence decreases.

(2) The rising slope of rectified and averaged EMG bursts for each motion coherence condition should reflect the drift rate, and should thus monotonically decrease as coherence decreases. (3) The proportion of trials featuring partial EMG bursts should increase as motion coherence decreases, and the latency of the first partial EMG burst should increase as motion coherence decreases as well. (4) The distributions of PMT and MT should exhibit a similar right-skewed shape for each subject and motion coherence level. (5) The between-trial correlation between PMT and MT should be null (without between-trial variability in drift rate) or slightly positive (with a modest amount of between-trial variability in drift rate, as classically assumed in the field of mathematical psychology). To maximize the probability of detecting each of these effects, we manipulated motion coherence in a random dot motion task and chose response buttons that require a relatively high amount of force (see Introduction). Our empirical results provide very strong evidence for each prediction. We next performed a quantitative assessment of DTDM by fitting the model to the joint distributions of PMT and MT in correct 
and error trials and to the correct and error choice probabilities simultaneously. DTDM provided a good fit to data, suggesting that it is a plausible processing account and thus generalizes to standard perceptual decision-making tasks.

Our findings also speak to the issue of selective influence. Smith and Lilbrun (2009) recently argued that apparent violations of selective influence should not be equated with a failure of the diffusion model as a whole, but should instead motivate theoretical extensions in order to better understand the underlying cause of these violations. We concur with Smith and Lilburn's view, and DTDM was specifically developed with this goal in mind (Servant et al., 2015). Without an integrated theory of decision-making and motor execution, the empirical observation that both mean PMT and mean MT increase as motion coherence decreases might have been interpreted as a severe violation of selective influence, and strong evidence against the DDM architecture. However, a simple theoretical extension of the model, motivated by the neurophysiology of the musculoskeletal motor system and recent insights from cognitive neuroscience (see Introduction), provides a straightforward account of several non-intuitive aspects of the data.

DTDM may also explain other empirical EMG observations in perceptual decisionmaking tasks. For example, recent cognitive neuroscience studies in both humans and nonhuman primates showed boosted sensory evidence representations under speed pressure, resulting in an increase in the rate of evidence accumulation compared to accuracy regimes (Heitz \& Schall, 2012; Reppert et al., 2018; Servant et al., 2019; Steinemann et al., 2018). Within DTDM, a larger drift rate in the speed compared to the accuracy condition would necessarily produce a faster mean MT and a steeper rising slope of averaged EMG bursts in the speed compared to the accuracy condition, which is exactly what has been observed by 
EMG studies (Spieser et al., 2017; Weindel et al., 2020; see Table 1). Conversely, manipulations of response bias are hypothesized to selectively modulate the starting point of evidence accumulation (Ratcliff \& McKoon, 2008; White \& Poldrack, 2014), so DTDM predicts no effect of response bias on MT. To the best of our knowledge, only one study analyzed the effect of response bias on EMG and reported no modulation of MT (Meckler et al., 2010; see Table 1), consistent with DTDM's prediction. A more challenging EMG finding concerns the effect of response force on PMT and MT. Two studies reported a larger MT for high compared to low response force conditions, and an opposite effect on PMT (Burle et al., 2002; Romaiguère et al., 1993; see Table 1). The effect of response force on MT is readily explained by a modulation of the distance between EMG and response thresholds within DTDM. However, the mechanism generating faster PMT in high compared to low response force conditions deserves additional investigation. Higher response force levels may increase arousal, which may in turn increase the amount of attentional resources allocated to the task and thus drift rate. Alternatively, subjects may decrease EMG thresholds under high force regimes to compensate for RT lengthening and maintain an optimal speed-accuracy balance. While these two hypotheses predict faster PMT in high compared to low force conditions, they make different predictions regarding accuracy and the rising slope of averaged EMG bursts. Specifically, the drift rate hypothesis predicts higher accuracy and a steeper rising slope in high compared to low force conditions. The EMG threshold hypothesis predicts a lower accuracy and no effect on the EMG rising slope in high compared to low force conditions. Consistent with the drift rate hypothesis, Burle et al. (2002) reported higher accuracy in high compared to low force conditions. However, Romaiguère et al. (1993) reported no significant effect of force on accuracy, suggesting that the two hypotheses may coexist. DTDM fits to data, coupled with an analysis of EMG rising slopes, should offer a strong test of these hypotheses. DTDM 
may thus provide new theoretical insight into a broad range of behavioral and EMG results in perceptual decision-tasks, and opens an exciting avenue for future research to better understand the relationship between decision-making and motor execution.

There are, of course, several pathways for model development. For example, DTDM could be combined with recent theoretical extensions of the DDM designed to account for how drift rate is computed, such as the integrated system model suggested by Smith and colleagues (Smith \& Lilburn, 2020; Smith \& Ratcliff, 2009). Another interesting avenue for future research concerns the mechanistic principles underlying the continuous flow of the decision variable up to M1. DTDM is agnostic with respect to these principles. It simply assumes a gate (EMG threshold) at the M1 level above which accumulated evidence is transmitted to the muscle. Verdonck et al. (2020) recently proposed a leaky integrated threshold architecture (LIT) that models the flow of information from perceptual evidence accumulation to the motor structures that prepare the response, such as M1. Specifically, LIT models perceptual evidence accumulation with a standard DDM, and assumes that motor preparation builds upon a leaky accumulation process that takes the accumulated evidence of the perceptual process as a continuous input. The dual-threshold assumption of DTDM could be combined with LIT in order to provide a full processing account of perceptual decisionmaking, motor preparation, and motor execution. Given the complexity of this model variant, we believe that a rigorous test requires simultaneous EMG and EEG recordings in order to provide empirical constraints on each processing level.

The present work provides a bridge between different fields of research. So far, motor execution has mainly been the object of movement sciences and biomechanics, and many psychologists consider that motor execution is not cognitively interesting. For example, Turner 
et al. (2015) developed a neural extension of the diffusion model that uses single-trial brain signals to better constrain model parameters. Motor execution is represented by a nondecision time parameter that "captures effects that are not cognitively interesting" ( $p$. 316). Our study speaks against this view, and demonstrates that motor execution -as quantified by EMG- offers a direct window on the decision variable. EMG thus appears particularly relevant to the fields of mathematical psychology and decision theory. However, we anticipate that the theoretical impact of DTDM will be much broader. For example, DTDM may provide new theoretical insights into studies of perception-action coupling (e.g., Witt, 2018) and movement disorders that appear to have a cognitive basis, such as paradoxical movements in Parkinson's disease (normalization of gait pattern when sensory cues are provided to guide the movement; Perugini et al., 2018). DTDM may also provide new insights into studies of impulse control disorders. This line of research classically distinguishes motoric versus decisional forms of impulsivity (Dalley \& Robbins, 2017), but DTDM suggests that these two seemingly different forms may have a common origin.

\section{Context of the Research}

The integrated theory of deciding and acting developed in this paper derives from two origins. The first being a popular French television quiz show from the 1990's which involved responding to trivia questions of varying difficulties by pressing a buzzer. The pressure applied to the buzzer appeared to depend on the difficulty of the decision and the participant's uncertainty: the more uncertain one was, the more hesitant hand pulses occurred (small

pressures on the buzzer, not strong enough to generate the rasping sound signaling a response), and the slower one pressed the buzzer. Up to now, no psychological theory was 
able to explain these motor phenomena that appeared to have a cognitive basis. The second source of influence concerns our previous EMG and computational work in conflict tasks (Servant et al., 2015), as explained in the Introduction section of the paper. Following this work, we hypothesized that motor phenomena observed in a variety of choice laboratory tasks should have a common processing origin. We then developed a research program aiming at testing this hypothesis and elucidating the processing mechanisms involved. The theory should shed light onto a variety of research problems that incorporate the motor system. One prominent example concerns movement disorders that appear to have a cognitive basis.

\section{References}

Afacan-Seref, K., Steinemann, N. A., Blangero, A., \& Kelly, S. P. (2018). Dynamic Interplay of Value and Sensory Information in High-Speed Decision Making. Current Biology: CB, 28(5), 795-802.e6. https://doi.org/10.1016/j.cub.2018.01.071

Arnold, N. R., Bröder, A., \& Bayen, U. J. (2015). Empirical validation of the diffusion model for recognition memory and a comparison of parameter-estimation methods. Psychological Research, 79(5), 882-898. https://doi.org/10.1007/s00426-014-0608-y

Bernhard, C. G., \& Bohm, E. (1954). Cortical representation and functional significance of the corticomotoneuronal system. A.M.A. Archives of Neurology and Psychiatry, 72(4), 473-502. https://doi.org/10.1001/archneurpsyc.1954.02330040075006

Boehm, U., Annis, J., Frank, M. J., Hawkins, G. E., Heathcote, A., Kellen, D., Krypotos, A.-M., Lerche, V., Logan, G. D., Palmeri, T. J., van Ravenzwaaij, D., Servant, M., Singmann, H., Starns, J. J., Voss, A., Wiecki, T. V., Matzke, D., \& Wagenmakers, E.-J. (2018). Estimating across-trial variability parameters of the Diffusion Decision Model: Expert advice and recommendations. Journal of Mathematical Psychology, 87, 46-75. https://doi.org/10.1016/j.jmp.2018.09.004 
Brown, S. D., \& Heathcote, A. (2008). The simplest complete model of choice response time: Linear ballistic accumulation. Cognitive Psychology, 57(3), 153-178. https://doi.org/10.1016/j.cogpsych.2007.12.002

Burle, B., \& Bonnet, M. (1999). What's an internal clock for?: From temporal information processing to temporal processing of information. Behavioural Processes, 45(1), 59-72. https://doi.org/10.1016/S0376-6357(99)00009-1

Burle, B., Possamaï, C.-A., Vidal, F., Bonnet, M., \& Hasbroucq, T. (2002). Executive control in the Simon effect: An electromyographic and distributional analysis. Psychological Research, 66(4), 324-336. https://doi.org/10.1007/s00426-002-0105-6

Cavanagh, P. R., \& Komi, P. V. (1979). Electromechanical delay in human skeletal muscle under concentric and eccentric contractions. European Journal of Applied Physiology and Occupational Physiology, 42(3), 159-163. https://doi.org/10.1007/BF00431022

Coles, M. G., Gratton, G., Bashore, T. R., Eriksen, C. W., \& Donchin, E. (1985). A psychophysiological investigation of the continuous flow model of human information processing. Journal of Experimental Psychology. Human Perception and Performance, 11(5), 529-553. https://doi.org/10.1037//0096-1523.11.5.529

Dalley, J. W., \& Robbins, T. W. (2017). Fractionating impulsivity: Neuropsychiatric implications. Nature Reviews. Neuroscience, 18(3), 158-171. https://doi.org/10.1038/nrn.2017.8 de Hollander, G., Labruna, L., Sellaro, R., Trutti, A., Colzato, L. S., Ratcliff, R., Ivry, R. B., \& Forstmann, B. U. (2016). Transcranial Direct Current Stimulation Does Not Influence the Speed-Accuracy Tradeoff in Perceptual Decision-making: Evidence from Three Independent Studies. Journal of Cognitive Neuroscience, 28(9), 1283-1294. https://doi.org/10.1162/jocn_a_00967

Disselhorst-Klug, C., Schmitz-Rode, T., \& Rau, G. (2009). Surface electromyography and muscle force: Limits in sEMG-force relationship and new approaches for applications. Clinical Biomechanics (Bristol, Avon), 24(3), 225-235. https://doi.org/10.1016/j.clinbiomech.2008.08.003 
Donkin, C., Brown, S., Heathcote, A., \& Wagenmakers, E.-J. (2011). Diffusion versus linear ballistic accumulation: Different models but the same conclusions about psychological processes? Psychonomic Bulletin \& Review, 18(1), 61-69. https://doi.org/10.3758/s13423-010-0022-4

Donner, T. H., Siegel, M., Fries, P., \& Engel, A. K. (2009). Buildup of choice-predictive activity in human motor cortex during perceptual decision making. Current Biology: $C B, 19(18), 1581-$ 1585. https://doi.org/10.1016/j.cub.2009.07.066

Dutilh, G., Annis, J., Brown, S. D., Cassey, P., Evans, N. J., Grasman, R. P. P. P., Hawkins, G. E., Heathcote, A., Holmes, W. R., Krypotos, A.-M., Kupitz, C. N., Leite, F. P., Lerche, V., Lin, Y.-S., Logan, G. D., Palmeri, T. J., Starns, J. J., Trueblood, J. S., van Maanen, L., ... Donkin, C. (2019). The Quality of Response Time Data Inference: A Blinded, Collaborative Assessment of the Validity of Cognitive Models. Psychonomic Bulletin \& Review, 26(4), 1051-1069. https://doi.org/10.3758/s13423-017-1417-2

Ebbesen, C. L., \& Brecht, M. (2017). Motor cortex-To act or not to act? Nature Reviews Neuroscience, 18(11), 694-705. https://doi.org/10.1038/nrn.2017.119

Evans, N. J. (2019). A method, framework, and tutorial for efficiently simulating models of decisionmaking. Behavior Research Methods, 51(5), 2390-2404. https://doi.org/10.3758/s13428019-01219-z

Evans, N. J. (2020). Think fast! The implications of emphasizing urgency in decision-making [Preprint]. PsyArXiv. https://doi.org/10.31234/osf.io/pfrb4

Forstmann, B. U., Ratcliff, R., \& Wagenmakers, E.-J. (2016). Sequential Sampling Models in Cognitive Neuroscience: Advantages, Applications, and Extensions. Annual Review of Psychology, 67, 641-666. https://doi.org/10.1146/annurev-psych-122414-033645

Gajdos, T., Fleming, S. M., Saez Garcia, M., Weindel, G., \& Davranche, K. (2019). Revealing subthreshold motor contributions to perceptual confidence. Neuroscience of Consciousness, 2019(1). https://doi.org/10.1093/nc/niz001 
Gold, J. I., \& Shadlen, M. N. (2007). The neural basis of decision making. Annual Review of Neuroscience, 30, 535-574. https://doi.org/10.1146/annurev.neuro.29.051605.113038

Gould, I. C., Nobre, A. C., Wyart, V., \& Rushworth, M. F. S. (2012). Effects of Decision Variables and Intraparietal Stimulation on Sensorimotor Oscillatory Activity in the Human Brain. The Journal of Neuroscience, 32(40), 13805-13818. https://doi.org/10.1523/JNEUROSCI.220012.2012

Gramfort, A., Luessi, M., Larson, E., Engemann, D. A., Strohmeier, D., Brodbeck, C., Goj, R., Jas, M., Brooks, T., Parkkonen, L., \& Hämäläinen, M. (2013). MEG and EEG data analysis with MNEPython. Frontiers in Neuroscience, 7, 267. https://doi.org/10.3389/fnins.2013.00267

Greenhouse, S. W., \& Geisser, S. (1959). On methods in the analysis of profile data. Psychometrika, 24(2), 95-112. https://doi.org/10.1007/BF02289823

Hasbroucq, T., Burle, B., Akamatsu, M., Vidal, F., \& Possamaï, C.-A. (2001). An electromyographic investigation of the effect of stimulus-response mapping on choice reaction time. Psychophysiology, 38(1), 157-162. https://doi.org/10.1017/\$0048577201001718

Hasbroucq, T., Burle, B., Vidal, F., \& Possamaï, C.-A. (2009). Stimulus-hand correspondence and direct response activation: An electromyographic analysis. Psychophysiology, 46(6), 1160-1169. https://doi.org/10.1111/j.1469-8986.2009.00865.x

Hasbroucq, T., Possamaï, C. A., Bonnet, B., \& Vidal, F. (1999). Effect of the irrelevant location of the response signal on choice reaction time: An electromyographic study in humans. Psychophysiology, 36(4), 522-526. https://doi.org/10.1017/s0048577299001602

Heathcote, A., \& Love, J. (2012). Linear Deterministic Accumulator Models of Simple Choice. Frontiers in Psychology, 3. https://doi.org/10.3389/fpsyg.2012.00292

Heitz, R. P., \& Schall, J. D. (2012). Neural Mechanisms of Speed-Accuracy Tradeoff. Neuron, 76(3), 616-628. https://doi.org/10.1016/j.neuron.2012.08.030 
Ho, T., Brown, S., van Maanen, L., Forstmann, B. U., Wagenmakers, E.-J., \& Serences, J. T. (2012). The Optimality of Sensory Processing during the Speed-Accuracy Tradeoff. The Journal of Neuroscience, 32(23), 7992-8003. https://doi.org/10.1523/JNEUROSCI.0340-12.2012

Huang, Y.-T., Georgiev, D., Foltynie, T., Limousin, P., Speekenbrink, M., \& Jahanshahi, M. (2015). Different effects of dopaminergic medication on perceptual decision-making in Parkinson's disease as a function of task difficulty and speed-accuracy instructions. Neuropsychologia, 75, 577-587. https://doi.org/10.1016/j.neuropsychologia.2015.07.012

Hübner, R., Steinhauser, M., \& Lehle, C. (2010). A dual-stage two-phase model of selective attention. Psychological Review, 117(3), 759-784. https://doi.org/10.1037/a0019471

Jeffreys, H. (1961). Theory of probability. Oxford University Press.

Kelly, S. P., \& O'Connell, R. G. (2013). Internal and External Influences on the Rate of Sensory Evidence Accumulation in the Human Brain. Journal of Neuroscience, 33(50), 19434-19441. https://doi.org/10.1523/JNEUROSCI.3355-13.2013

Lacourpaille, L., Hug, F., \& Nordez, A. (2013). Influence of Passive Muscle Tension on Electromechanical Delay in Humans. PLOS ONE, 8(1), e53159. https://doi.org/10.1371/journal.pone.0053159

Lemon, R. N. (2008). Descending pathways in motor control. Annual Review of Neuroscience, 31, 195-218. https://doi.org/10.1146/annurev.neuro.31.060407.125547

Lerche, V., \& Voss, A. (2017). Retest reliability of the parameters of the Ratcliff diffusion model. Psychological Research, 81(3), 629-652. https://doi.org/10.1007/s00426-016-0770-5 Liu, J., \& Liu, Q. (2016). Use of the integrated profile for voluntary muscle activity detection using EMG signals with spurious background spikes: A study with incomplete spinal cord injury. Biomedical Signal Processing and Control, 24, 19-24. https://doi.org/10.1016/j.bspc.2015.09.004 Mauchly, J. W. (1940). Significance Test for Sphericity of a Normal n-Variate Distribution. The Annals of Mathematical Statistics, 11(2), 204-209. JSTOR. 
Meckler, C., Allain, S., Carbonnell, L., Hasbroucq, T., Burle, B., \& Vidal, F. (2010). Motor inhibition and response expectancy: A Laplacian ERP study. Biological Psychology, 85(3), 386-392. https://doi.org/10.1016/j.biopsycho.2010.08.011

Miller, J., Ulrich, R., \& Rinkenauer, G. (1999). Effects of stimulus intensity on the lateralized readiness potential. Journal of Experimental Psychology: Human Perception and Performance, 25(5), 1454-1471. https://doi.org/10.1037/0096-1523.25.5.1454

Nelder, J. A., \& Mead, R. (1965). A Simplex Method for Function Minimization. The Computer Journal, 7(4), 308-313. https://doi.org/10.1093/comjnl/7.4.308

Nosofsky, R. M., \& Palmeri, T. J. (1997). An exemplar-based random walk model of speeded classification. Psychological Review, 104(2), 266-300. https://doi.org/10.1037/0033295X.104.2.266

O'Connell, R. G., Dockree, P. M., \& Kelly, S. P. (2012). A supramodal accumulation-to-bound signal that determines perceptual decisions in humans. Nature Neuroscience, 15(12), 1729-1735. https://doi.org/10.1038/nn.3248

O'Connell, R. G., Shadlen, M. N., Wong-Lin, K., \& Kelly, S. P. (2018). Bridging Neural and Computational Viewpoints on Perceptual Decision-Making. Trends in Neurosciences, 41(11), 838-852. https://doi.org/10.1016/j.tins.2018.06.005

Peirce, J., Gray, J. R., Simpson, S., MacAskill, M., Höchenberger, R., Sogo, H., Kastman, E., \& Lindeløv, J. K. (2019). PsychoPy2: Experiments in behavior made easy. Behavior Research Methods, 51(1), 195-203. https://doi.org/10.3758/s13428-018-01193-y

Perry, J., \& Bekey, G. A. (1981). EMG-force relationships in skeletal muscle. Critical Reviews in Biomedical Engineering, 7(1), 1-22.

Perugini, A., Ditterich, J., Shaikh, A. G., Knowlton, B. J., \& Basso, M. A. (2018). Paradoxical DecisionMaking: A Framework for Understanding Cognition in Parkinson's Disease. Trends in Neurosciences, 41(8), 512-525. https://doi.org/10.1016/j.tins.2018.04.006 
Pilly, P. K., \& Seitz, A. R. (2009). What a difference a parameter makes: A psychophysical comparison of random dot motion algorithms. Vision Research, 49(13), 1599-1612. https://doi.org/10.1016/j.visres.2009.03.019

Rae, B., Heathcote, A., Donkin, C., Averell, L., \& Brown, S. (2014). The hare and the tortoise: Emphasizing speed can change the evidence used to make decisions. Journal of Experimental Psychology. Learning, Memory, and Cognition, 40(5), 1226-1243.

https://doi.org/10.1037/a0036801

Ratcliff, R. (1978). A theory of memory retrieval. Psychol. Rev, 85(2), 59-108.

Ratcliff, R. (1979). Group reaction time distributions and an analysis of distribution statistics. Psychological Bulletin, 86(3), 446-461. https://doi.org/10.1037/0033-2909.86.3.446

Ratcliff, R., \& McKoon, G. (2008). The Diffusion Decision Model: Theory and Data for Two-Choice Decision Tasks. Neural Computation, 20(4), 873-922. https://doi.org/10.1162/neco.2008.12$06-420$

Ratcliff, R., \& Rouder, J. N. (1998). Modeling Response Times for Two-Choice Decisions. Psychological Science, 9(5), 347-356. JSTOR.

Ratcliff, R., \& Smith, P. L. (2004). A comparison of sequential sampling models for two-choice reaction time. Psychological Review, 111(2), 333-367. https://doi.org/10.1037/0033295X.111.2.333

Ratcliff, R., Smith, P. L., Brown, S. D., \& McKoon, G. (2016). Diffusion Decision Model: Current Issues and History. Trends in Cognitive Sciences, 20(4), 260-281. https://doi.org/10.1016/j.tics.2016.01.007

Ratcliff, R., \& Tuerlinckx, F. (2002). Estimating parameters of the diffusion model: Approaches to dealing with contaminant reaction times and parameter variability. Psychonomic Bulletin \& Review, 9(3), 438-481. https://doi.org/10.3758/BF03196302

Reppert, T. R., Servant, M., Heitz, R. P., \& Schall, J. D. (2018). Neural mechanisms of speed-accuracy tradeoff of visual search: Saccade vigor, the origin of targeting errors, and comparison of the 
superior colliculus and frontal eye field. Journal of Neurophysiology, 120(1), 372-384. https://doi.org/10.1152/jn.00887.2017

Rochet, N., Spieser, L., Casini, L., Hasbroucq, T., \& Burle, B. (2014). Detecting and correcting partial errors: Evidence for efficient control without conscious access. Cognitive, Affective \& Behavioral Neuroscience, 14(3), 970-982. https://doi.org/10.3758/s13415-013-0232-0

Romaiguère, P., Hasbroucq, T., Possamaï, C. A., \& Seal, J. (1993). Intensity to force translation: A new effect of stimulus-response compatibility revealed by analysis of response time and electromyographic activity of a prime mover. Brain Research. Cognitive Brain Research, 1(3), 197-201. https://doi.org/10.1016/0926-6410(93)90028-4

Santello, M., \& McDonagh, M. J. (1998). The control of timing and amplitude of EMG activity in landing movements in humans. Experimental Physiology, 83(6), 857-874. https://doi.org/10.1113/expphysiol.1998.sp004165

Selen, L. P. J., Shadlen, M. N., \& Wolpert, D. M. (2012). Deliberation in the motor system: Reflex gains track evolving evidence leading to a decision. The Journal of Neuroscience: The Official Journal of the Society for Neuroscience, 32(7), 2276-2286. https://doi.org/10.1523/JNEUROSCI.5273-11.2012

Servant, M. (2021, January 8). An integrated theory of deciding and acting. https://doi.org/10.17605/OSF.IO/R5C2B

Servant, M., Tillman, G., Schall, J. D., Logan, G. D., \& Palmeri, T. J. (2019). Neurally constrained modeling of speed-accuracy tradeoff during visual search: Gated accumulation of modulated evidence. Journal of Neurophysiology, 121(4), 1300-1314. https://doi.org/10.1152/jn.00507.2018

Servant, M., van Wouwe, N., Wylie, S. A., \& Logan, G. D. (2018). A model-based quantification of action control deficits in Parkinson's disease. Neuropsychologia, 111, 26-35. https://doi.org/10.1016/j.neuropsychologia.2018.01.014 
Servant, M., White, C., Montagnini, A., \& Burle, B. (2015). Using Covert Response Activation to Test Latent Assumptions of Formal Decision-Making Models in Humans. The Journal of Neuroscience: The Official Journal of the Society for Neuroscience, 35(28), 10371-10385. https://doi.org/10.1523/JNEUROSCI.0078-15.2015

Servant, M., White, C., Montagnini, A., \& Burle, B. (2016). Linking Theoretical Decision-making Mechanisms in the Simon Task with Electrophysiological Data: A Model-based Neuroscience Study in Humans. Journal of Cognitive Neuroscience, 28(10), 1501-1521. https://doi.org/10.1162/jocn_a_00989

Smid, H. G., Mulder, G., \& Mulder, L. J. (1990). Selective response activation can begin before stimulus recognition is complete: A psychophysiological and error analysis of continuous flow. Acta Psychologica, 74(2-3), 169-201. https://doi.org/10.1016/0001-6918(90)90005-z

Smith, P. L., \& Lilburn, S. D. (2020). Vision for the blind: Visual psychophysics and blinded inference for decision models. Psychonomic Bulletin \& Review. https://doi.org/10.3758/s13423-02001742-7

Smith, P. L., \& Ratcliff, R. (2009). An integrated theory of attention and decision making in visual signal detection. Psychological Review, 116(2), 283-317. https://doi.org/10.1037/a0015156

Spieser, L., \& Burle, B. (in preparation). Debut-Detecting Emg BUrsT - a python package to process and detect signal burst(s) onset and offset.

Spieser, L., Servant, M., Hasbroucq, T., \& Burle, B. (2017). Beyond decision! Motor contribution to speed-accuracy trade-off in decision-making. Psychonomic Bulletin \& Review, 24(3), 950-956. https://doi.org/10.3758/s13423-016-1172-9

Stålberg, E., van Dijk, H., Falck, B., Kimura, J., Neuwirth, C., Pitt, M., Podnar, S., Rubin, D. I., Rutkove, S., Sanders, D. B., Sonoo, M., Tankisi, H., \& Zwarts, M. (2019). Standards for quantification of EMG and neurography. Clinical Neurophysiology: Official Journal of the International Federation of Clinical Neurophysiology, 130(9), 1688-1729. https://doi.org/10.1016/j.clinph.2019.05.008 
Starns, J. J., Ratcliff, R., \& White, C. N. (2012). Diffusion model drift rates can be influenced by decision processes: An analysis of the strength-based mirror effect. Journal of Experimental Psychology. Learning, Memory, and Cognition, 38(5), 1137-1151. https://doi.org/10.1037/a0028151

Steinemann, N. A., O'Connell, R. G., \& Kelly, S. P. (2018). Decisions are expedited through multiple neural adjustments spanning the sensorimotor hierarchy. Nature Communications, 9(1), 3627. https://doi.org/10.1038/s41467-018-06117-0

Storn, R., \& Price, K. (1997). Differential Evolution - A Simple and Efficient Heuristic for global Optimization over Continuous Spaces. Journal of Global Optimization, 11(4), 341-359. https://doi.org/10.1023/A:1008202821328

Turner, B. M., van Maanen, L., \& Forstmann, B. U. (2015). Informing cognitive abstractions through neuroimaging: The neural drift diffusion model. Psychological Review, 122(2), 312-336. https://doi.org/10.1037/a0038894

Ulrich, R., Schröter, H., Leuthold, H., \& Birngruber, T. (2015). Automatic and controlled stimulus processing in conflict tasks: Superimposed diffusion processes and delta functions. Cognitive Psychology, 78, 148-174. https://doi.org/10.1016/j.cogpsych.2015.02.005

Usher, M., \& McClelland, J. L. (2001). The time course of perceptual choice: The leaky, competing accumulator model. Psychological Review, 108(3), 550-592. https://doi.org/10.1037/0033$295 x .108 .3 .550$

van Doorn, J., van den Bergh, D., Böhm, U., Dablander, F., Derks, K., Draws, T., Etz, A., Evans, N. J., Gronau, Q. F., Haaf, J. M., Hinne, M., Kucharský, Š., Ly, A., Marsman, M., Matzke, D., Gupta, A. R. K. N., Sarafoglou, A., Stefan, A., Voelkel, J. G., \& Wagenmakers, E.-J. (2020). The JASP guidelines for conducting and reporting a Bayesian analysis. Psychonomic Bulletin \& Review. https://doi.org/10.3758/s13423-020-01798-5 
Verdonck, S., Loossens, T., \& Philiastides, M. G. (2020). The Leaky Integrating Threshold and its impact on evidence accumulation models of choice response time (RT). Psychological Review. https://doi.org/10.1037/rev0000258

Vigotsky, A. D., Halperin, I., Lehman, G. J., Trajano, G. S., \& Vieira, T. M. (2018). Interpreting Signal Amplitudes in Surface Electromyography Studies in Sport and Rehabilitation Sciences. Frontiers in Physiology, 8. https://doi.org/10.3389/fphys.2017.00985

Voss, A., Rothermund, K., \& Voss, J. (2004). Interpreting the parameters of the diffusion model: An empirical validation. Memory \& Cognition, 32(7), 1206-1220. https://doi.org/10.3758/BF03196893

Weindel, G., Anders, R., Alario, F.-X., \& Burle, B. (2020). Assessing model-based inferences in decision making with single-trial response time decomposition. https://doi.org/10.31234/osf.io/kc26f

White, C. N., \& Poldrack, R. A. (2014). Decomposing bias in different types of simple decisions. Journal of Experimental Psychology. Learning, Memory, and Cognition, 40(2), 385-398. https://doi.org/10.1037/a0034851

White, C. N., Ratcliff, R., \& Starns, J. J. (2011). Diffusion models of the flanker task: Discrete versus gradual attentional selection. Cognitive Psychology, 63(4), 210-238. https://doi.org/10.1016/j.cogpsych.2011.08.001

Witt, J. K. (2018). Perception and action. In Stevens' handbook of experimental psychology and cognitive neuroscience: Vol. 2: Sensation, Perception, and Attention (Wixted, J. T., pp. 489523). Wiley.

Wyart, V., de Gardelle, V., Scholl, J., \& Summerfield, C. (2012). Rhythmic Fluctuations in Evidence Accumulation during Decision Making in the Human Brain. Neuron, 76(4), 847-858. https://doi.org/10.1016/j.neuron.2012.09.015 